\title{
Existence of Three Solutions for Nonlinear Operator Equations and Applications to Second-Order Differential Equations
}

\author{
Mingliang Song (D) and Shuyuan Mei \\ Mathematics and Information Technology School, Jiangsu Second Normal University, Nanjing 210013, China \\ Correspondence should be addressed to Mingliang Song; mlsong2004@163.com
}

Received 18 December 2020; Revised 6 January 2021; Accepted 9 January 2021; Published 29 January 2021

Academic Editor: Maria Alessandra Ragusa

Copyright (c) 2021 Mingliang Song and Shuyuan Mei. This is an open access article distributed under the Creative Commons Attribution License, which permits unrestricted use, distribution, and reproduction in any medium, provided the original work is properly cited.

\begin{abstract}
The existence of three solutions for nonlinear operator equations is established via index theory for linear self-adjoint operator equations, critical point reduction method, and three critical points theorems obtained by Brezis-Nirenberg, Ricceri, and Averna-Bonanno. Applying the results to second-order Hamiltonian systems satisfying generalized periodic boundary conditions or Sturm-Liouville boundary conditions and elliptic partial differential equations satisfying Dirichlet boundary value conditions, we obtain some new theorems concerning the existence of three solutions.
\end{abstract}

\section{Introduction and Main Results}

In their excellent paper [1] in 1991, Brezis-Nirenberg proved the existence of two nonzero critical points under suitable assumptions by a negative gradient flow and linking. The obtained result was applied to second-order Hamiltonian systems and yielded the existence of three periodic solutions for these systems. After that, in [2], Tang obtained one existence of at least three periodic solutions for second-order Hamiltonian systems by using two nonzero critical points theorem in [1]. This result generalizes the corresponding result in [1]. In addition, in [3], one new existence of at least three periodic solutions for second-order Hamiltonian systems was obtained by using the critical point reduction method and two nonzero critical points theorem in $[1,4]$.

Moreover, after the three critical points theorem (see [5, 6]) of Ricceri appearing, it has proved to be one of the most often widely used to solve differential equations, such as elliptic partial differential equations satisfying Dirichlet boundary value conditions (see [7-12]) and second-order Hamiltonian systems satisfying periodic boundary value conditions (see $[3,13])$. Recently, a general three critical points theorem is given by Averna-Bonanno [14]. After that, in [15], the existence of at least three periodic solutions was established for a class of $p$-Hamiltonian systems by two general three critical points theorems in $[6,14]$.

It is well known that the above-mentioned problems can be displayed as a self-adjoint operator equation. For the multiplicity of self-adjoint operator equation, Chang proved the existence of three distinct solutions under suitable assumptions via Morse theory in his excellent paper [16] in 1981. The obtained results were applied to second-order Hamiltonian systems and wave equations and yielded the existence of three periodic solutions for these systems. Similar results can be found in [17-21].

Inspired by the ideas of $[16,18,19]$, in this paper, we reconsider in the framework of the operator equations some theorems proved in $[1-3,9,10,15,22]$ and generalize these theorems to nonlinear self-adjoint operator equations. Our technical approach is based on some three critical points theorems obtained by Brezis-Nirenberg [1], Averna-Bonanno [14], and Ricceri [6] or the critical point reduction method in [4]. As applications, we consider the existence of three solutions for second-order Hamiltonian systems satisfying generalized periodic boundary conditions or SturmLiouville boundary conditions and elliptic partial differential equations satisfying Dirichlet boundary value conditions. Meanwhile, we give some examples (see Examples 1-4 in 
Section 4.1) to illustrate the effectiveness of our results and point out that our results improve and generalize the corresponding results in $[1-3,9,10,15,22]$ via several remarks (see Remarks 18, 20, 22, 24, 29, and 33 in Section 4).

Before expressing our main results, for the convenience of the readers, we give a brief introduction to the index theory and the setting of the space; for some details, we refer to [23, 24] (or see Section 2).

Let $X$ be a real infinite-dimensional separable Hilbert space with inner product $(\cdot, \cdot)_{X}$ and the corresponding norm $\|\cdot\|_{X}$. Let $A: D(A) \subset X \longrightarrow X$ be an unbounded linear selfadjoint operator with $\sigma(A)=\sigma_{d}(A)$ bounded from below. This implies that there is an orthonormal basis $\left\{e_{j}\right\}_{j=1}^{\infty}$ of $X$ and $\lambda_{1} \leq \lambda_{2} \leq \cdots$ such that $A e_{j}=\lambda_{j} e_{j}, D(A)=\left\{\sum_{j=1}^{\infty} c_{j} e_{j} \mid \sum_{j=1}^{\infty}\right.$ $\left.\lambda_{j}^{2} c_{j}^{2}<\infty\right\}$. Meanwhile, let $Z \equiv D\left(|A|^{1 / 2}\right)=\left\{\sum_{j=1}^{\infty} c_{j} e_{j}\left|\sum_{j=1}^{\infty}\right| \lambda_{j} \mid\right.$ $\left.c_{j}^{2}<\infty\right\}$ with the norm $\|x\|_{Z}^{2}=\sum_{j=1}^{\infty}\left(1+\left|\lambda_{j}\right|\right) c_{j}^{2}$. For any $x=$ $\sum_{j=1}^{\infty} c_{j} e_{j} \in Z, y=\sum_{j=1}^{\infty} d_{j} e_{j} \in Z$, we define a bilinear form

$$
a(x, y)=\sum_{j=1}^{\infty} \lambda_{j} c_{j} d_{j} .
$$

By $(A x, y)_{X}=a(x, y)$ if $x \in D(A), y \in Z$, we can see that $a(x, y)$ is the extension of $(A x, y)_{X}$ on $Z$. Moreover, let $B \in$ $L_{s}(X)$ be the usual space consisting of bounded symmetric operators in $X$. For any $B \in L_{s}(X)$, we define

$$
\begin{aligned}
& v_{A}(B)=\operatorname{dimker}(A-B), \\
& i_{A}(B)=\sum_{\lambda<0} v_{A}(B+\lambda I d),
\end{aligned}
$$

which are introduced by Dong (see Definition 3.1.1 and Proposition 3.1.4 in [23] or Definition 7.1.1 in [24]).

Let us consider the following two nonlinear operator equations:

$$
\begin{gathered}
A x-B_{0} x=\nabla \Phi(x), \\
A x-B_{0} x+B_{1} x=\eta \nabla \Phi(x)+\mu \nabla \Psi(x),
\end{gathered}
$$

where $\eta, \mu \in[0,+\infty), B_{0}, B_{1} \in L_{s}(X)$ with $i_{A}\left(B_{0}\right)=0, v_{A}\left(B_{0}\right)$ $\neq 0$, and $B_{1}$ is positive definite for all $x \in X$, that is, there exists a positive constant $\underline{\lambda}$ such that $\left(B_{1} x, x\right)_{X} \geq \underline{\lambda}\|x\|_{X}^{2}$ for all $x \in X$. Meanwhile, $\Phi$ and $\Psi$ satisfy, respectively.

$\left(\Phi_{0}\right) \Phi \in C^{1}(Z, R)$ is weakly continuous with compact derivative, that is, $x_{n} \rightarrow x_{0}$ in $Z$ implies that $\Phi\left(x_{n}\right) \longrightarrow \Phi\left(x_{0}\right)$ and $\Phi^{\prime}\left(x_{n}\right) \longrightarrow \Phi^{\prime}\left(x_{0}\right)$. Moreover, for every $x \in Z$, there exists $\nabla \Phi(x) \in X$ such that $\Phi^{\prime}(x) y=(\nabla \Phi(x), y)_{X}$ for all $y \in Z$.

$\left(\Psi_{0}\right) \Psi \in C^{1}(Z, R)$ is weakly continuous with compact derivative. Moreover, for every $x \in Z$, there exists $\nabla \Psi(x) \in$ $X$ such that $\Psi^{\prime}(x) y=(\nabla \Psi(x), y)_{X}$ for all $y \in Z$.

Noticing that $i_{A}\left(B_{0}\right)=0, v_{A}\left(B_{0}\right) \neq 0$, we have $\min \sigma(A$ $\left.-B_{0}\right)=0$. Then, we can define another inner product

$$
\langle x, y\rangle=a(x, y)-\left(B_{0} x, y\right)_{X}+(x, y)_{X}, \quad \forall x, y \in Z,
$$

with the corresponding norm

$$
\|x\|=\left(a(x, x)-\left(B_{0} x, x\right)_{X}+\|x\|_{X}^{2}\right)^{1 / 2}, \quad \forall x \in Z .
$$

Clearly, $\|\cdot\|$ is equivalent to $\|\cdot\|_{Z}$.

Let

$$
\|x\|_{B_{1}}=\left(a(x, x)-\left(B_{0} x, x\right)_{X}+\right)^{1 / 2}, \quad \forall x \in Z .
$$

Since $B_{1}$ is positive definite, there exist $\bar{\lambda} \geq \underline{\lambda}>0$ such that $\bar{\lambda}\|x\|_{X}^{2} \geq\left(B_{1} x, x\right)_{X} \geq \underline{\lambda}\|x\|_{X}^{2}$ for all $x \in X$. So we have

$$
\min \{1, \underline{\lambda}\}\|x\|^{2} \leq\|x\|_{B_{1}}^{2} \leq \max \{1, \bar{\lambda}\}\|x\|^{2} .
$$

Observe that the embedding $Z \hookrightarrow X$ is compact. Further, assume that there exists a Banach space $X_{0}$ satisfying $Z \subset$ $X_{0} \subset X$ and the embeddings $Z \hookrightarrow X_{0}$ and $X_{0} \hookrightarrow X$ are compact and continuous, respectively. Thus, there exists $k_{1}>0$ such that $\|x\|_{X_{0}} \leq k_{1}\|x\|$ for all $x \in Z$. Let

$$
k_{2}=\sup _{x \in Z \backslash\{\theta\}} \frac{\|x\|_{X_{0}}}{\|x\|_{B_{1}}} .
$$

From (8), we have

$$
0<k_{2} \leq \tilde{k}=k_{1}(\min \{1, \underline{\lambda}\})^{-1 / 2} .
$$

Let $X_{1}$ be a nontrivial subspace of $X$. For $B_{1}, B_{2} \in L_{s}(X)$, we write $B_{1} \leq B_{2}$ with respect to $X_{1}$ if and only if $\left(B_{1} x, x\right)_{X}$ $\leq\left(B_{2} x, x\right)_{X}$ for all $x \in X_{1}$; we write $B_{1}<B_{2}$ w.r.t. $X_{1}$ if and only if $\left(B_{1} x, x\right)_{X}<\left(B_{2} x, x\right)_{X}$ for all $x \in X_{1} \backslash\{\theta\}$. If $X_{1}=X$, then we just write $B_{1} \leq B_{2}$ or $B_{1}<B_{2}$.

Next, we use the index $\left(i_{A}(B), v_{A}(B)\right) \in N \times N$ defined in $[23,24]$ (see Section 2) for all $B \in L_{s}(X)$ to reach our main results.

Theorem 1. Assume that $\Phi$ satisfies $\left(\Phi_{0}\right)$ and

$\left(\Phi_{1}\right)$ there exist $c_{0}>0$ and $B_{2} \in \mathscr{L}_{s}(X)$ with $B_{2} \geq 0, v_{A}$ $\left(B_{0}+B_{2}\right)=0$ and $i_{A}\left(B_{0}+B_{2}\right)=v_{A}\left(B_{0}\right)$, such that

$$
\left|\left(\nabla \Phi(x), x_{X}\right)\right| \leq\left(B_{2} x, x\right)_{X}+c_{0}\|x\|,
$$

for all $x \in X$

$\left(\Phi_{2}\right)$ there exists a positive definite $B_{3} \in \mathscr{L}_{s}(X)$ such that $\Phi(x)-(1 / 2)\left(B_{3} x, x\right)_{X}$ is convex

Then, problem (3) has at least one solution with saddle character in $Z$ (i.e., the solution is a saddle point).

Assume in addition that

$\left(\Phi_{3}\right)$ there exist $\delta>0, B_{01}, B_{02} \in \mathscr{L}_{s}(X)$ with $B_{02}>B_{01}$ $>B_{0}, \quad v_{A}\left(B_{0 i}\right) \neq 0(i=1,2)$ and $i_{A}\left(B_{02}\right)=i_{A}\left(B_{01}\right)+v_{A}\left(B_{01}\right)$, such that

$$
\frac{1}{2}\left(\left(B_{01}-B_{0}\right) x, x\right)_{X} \leq \Phi(x) \leq \frac{1}{2}\left(\left(B_{02}-B_{0}\right) x, x\right)_{X}
$$

for all $\|x\| \leq \delta$. Then, problem (3) has at least three distinct solutions in $Z$. 
Theorem 2. Assume that $\Phi$ satisfies $\left(\Phi_{0}\right)$ and

$\left(\Phi_{4}\right)$ there exist $c_{1}, c_{2}>0$ and $\gamma_{0} \in(0,1)$ such that

$$
\left|(\nabla \Phi(x), y)_{X}\right| \leq c_{1}\|x\|_{X_{0}}^{\gamma_{0}} \cdot\|y\|_{X_{0}}+c_{2}\|y\|_{X_{0}},
$$

for all $x, y \in X$

$\left(\Phi_{5}\right)$ there exists $c_{3}>\left(c_{1}^{2} k_{1}^{2} / 2 \varepsilon_{2}^{2}\right)>0$ such that

$$
\limsup _{\|x\| \rightarrow+\infty} \frac{\Phi(x)}{\|x\|^{2 \gamma_{0}}}<-c_{3}
$$

for all $x \in \operatorname{ker}\left(A-B_{0}\right)$, where the constants $k_{1}$ and $\varepsilon_{2}$ are given by (9) and (49), respectively.

Then, problem (3) has at least one solution in Z. Further, assume that $\Phi$ satisfies $\left(\Phi_{3}\right)$. Then, problem (3) has at least three distinct solutions in $Z$.

Theorem 3. Assume that $\Phi$ satisfies $\left(\Phi_{0}\right)$ and

$\left(\Phi_{6}\right) \Phi(\theta)=0$

$\left(\Phi_{7}\right)$ there exist $c_{3}, c_{4} \in R^{+}$and $\gamma \in(0,2)$ such that $\Phi(x)$ $\leq c_{3}\|x\|_{X_{0}}^{\gamma}+c_{4}$ for all $x \in Z$

$\left(\Phi_{8}\right)$ there exist a positive constant $\tilde{c}$ and a $x_{0} \in$ ker $\left(A-B_{0}\right)$ with $\tilde{c}<\tilde{k}\left\|x_{0}\right\|_{X} \sqrt{\underline{\lambda}}$, such that

$$
\sup _{x \in E} \Phi(x)<\frac{\tilde{c}^{2}}{\bar{\lambda} \tilde{k}^{2}\left\|x_{0}\right\|_{X}^{2}} \Phi\left(x_{0}\right),
$$

where $E=\left\{x \in Z \mid\|x\|_{X_{0}} \leq \tilde{c}\right\}$.

Then, there exist an open interval $\Lambda \subseteq[0,+\infty)$ and a positive number $r_{0}$ with the following property: for each $\eta \in \Lambda$ and for every $\Psi: X \longrightarrow R$ satisfies $\left(\Psi_{0}\right)$, there exists $\rho>0$ such that, for each $\mu \in[0, \rho]$, problem (4) has at least three distinct solutions whose norms in $Z$ are less than $r_{0}$.

Theorem 4. Assume that $x_{0}, c_{3}, c_{4}, \tilde{c}, \gamma$, and $E$ are the same as in Theorem 3, $\Phi$ satisfies $\left(\Phi_{0}\right),\left(\Phi_{6}\right),\left(\Phi_{7}\right)$, and $\left(\Phi_{9}\right)$

$$
\sup _{x \in E} \Phi(x)<\frac{\tilde{c}^{2}}{\tilde{c}^{2}+\bar{\lambda} \tilde{k}^{2}\left\|x_{0}\right\|_{X}^{2}} \Phi\left(x_{0}\right) .
$$

Then, for each

$$
\eta \in\left(\frac{\bar{\lambda}\left\|x_{0}\right\|_{X}^{2}}{2\left(\Phi\left(x_{0}\right)-\sup _{x \in E} \Phi(x)\right)}, \frac{\tilde{c}^{2}}{2 \tilde{k}^{2} \sup _{x \in E} \Phi(x)}\right)
$$

problem (4) admits at least three distinct solutions provided $\mu=0$.

Remark 5. For some examples of the scalar equations corresponding to Theorems 1-4, we will give in Section 4, respectively.

The paper will be organized as follows. In Section 2, we recall first a saddle point reduction theorem under rather general assumptions in [4], a general two nonzero critical points theorem in [1], and two general three critical points theorems in $[6,14]$ as well as one proposition in [25]. Then, we recall some useful conclusions of index theory for linear self-adjoint operator equations from [23, 24]. Finally, we quote a lemma in [26], which verifies that (3) and (4) possess a variational construction in $Z$. In Section 3, we prove Theorems 1-4. In Section 4, we will investigate its applications to second-order Hamiltonian systems satisfying generalized periodic boundary conditions or Sturm-Liouville boundary conditions and second-order elliptic partial differential equations satisfying Dirichlet boundary value conditions. Meanwhile, we give some examples and some remarks to illustrate that the corresponding results of $[1-3,9,10,15$, 22] are special cases of these results in a sense.

\section{Preliminaries}

In order to prove our main results, we recall first four lemmas and one proposition due to $[1,4,6,14,25]$, respectively.

Lemma 6 (see [4], Theorem 2.3). Let $Z_{1}, Z_{2}$ be Hilbert spaces, and let $\psi \in C^{1}\left(Z_{1} \times Z_{2}, R\right)$. Suppose that $\psi$ satisfies $-D_{1} \psi$ $\left(\cdot, x_{2}\right): Z_{1} \longrightarrow Z_{1}$ is $\alpha$-monotone for all $x_{2} \in Z_{2}$, that is, there exists $\alpha>0$ such that

$$
\begin{aligned}
& \left\langle-D_{1} \psi\left(x_{1,1}, x_{2}\right)+D_{1} \psi\left(x_{1,2}, x_{2}\right), x_{1,1}-x_{1,2}\right\rangle \\
& \geq \alpha\left\|x_{1,1}-x_{1,2}\right\|^{2} \forall x_{1,1}, x_{1,2} \in Z_{1} .
\end{aligned}
$$

Then, there exists a map $\phi \in C\left(Z_{2}, Z_{1}\right)$ such that $\phi\left(x_{2}\right)$ $=x_{1}\left(x_{2}\right)$ is the unique saddle point of $\psi\left(\cdot, x_{2}\right)$ for every $x_{2}$ $\in Z_{2}$. Moreover, the map $\varphi: Z_{2} \longrightarrow R$ defined by

$$
\varphi\left(x_{2}\right)=\psi\left(x_{1}\left(x_{2}\right), x_{2}\right)=\sup _{x_{1} \in Z_{1}} \psi\left(x_{1}, x_{2}\right)
$$

is continuously differentiable, and its derivative is given by

$$
\varphi^{\prime}\left(x_{2}\right)=D_{2} \psi\left(x_{1}\left(x_{2}\right), x_{2}\right) \text { for every } x_{2} \in Z_{2} \text {. }
$$

Lemma 7 (see [1], Theorem 4). Let $Z$ be a reflexive real Banach space with a direct sum decomposition $Z=Z_{1} \oplus Z_{2}$ and $\operatorname{dim} Z_{1}<+\infty$. Let $I \in C^{1}(Z, R)$ with $I(\theta)=0$, satisfying (PS) condition and assume that $I$ is bounded below and $\inf _{Z} I<0$. Assume also that there is $\delta>0$ such that

$$
\begin{cases}I(x) \leq 0, & \text { for } x \in Z_{1},\|x\| \leq \delta \\ I(x) \geq 0, & \text { for } x \in Z_{2},\|x\| \leq \delta\end{cases}
$$

Then, I has at least two nonzero critical points.

Lemma 8 (see [6], Theorem 1). Let $Z$ be a reflexive real Banach space, $\tilde{I} \subseteq R$ an interval, $J: Z \longrightarrow R$ a sequentially weakly lower semicontinuous $C^{1}$ functional whose derivative admits a continuous inverse on $Z^{*}$, and $J$ bounded on each bounded subset of $Z, \Phi: Z \longrightarrow R$ a $C^{1}$ functional with 
compact derivative. Assume that

$$
\lim _{\|x\| \rightarrow+\infty}(J(x)-\eta \Phi(x))=+\infty
$$

for all $\eta \in \tilde{I}$ and that there exists $\beta \in R$ such that

$$
\sup _{\eta \in \tilde{I}} \inf _{x \in Z}(J(x)+\eta(\beta-\Phi(x)))<\inf _{x \in Z} \sup _{\eta \in \tilde{I}}(J(x)+\eta(\beta-\Phi(x))) .
$$

Then, there exist a nonempty open set $\Lambda \in \tilde{I}$ and a positive number $r_{0}$ with the following property: for every $\eta \in \Lambda$ and every $C^{1}$ functional $\Psi: Z \longrightarrow R$ with compact derivative, there exists $\rho>0$ such that, for each $\mu \in[0, \rho]$, the equation

$$
J^{\prime}(x)-\eta \Phi^{\prime}(x)-\mu \Psi^{\prime}(x)=0
$$

has at least three solutions whose norms in $Z$ are less than $r_{0}$.

Proposition 9 (see [25], Proposition 3.1). Let $Z$ be a nonempty set and $J, \Phi$ two real functions on $Z$. Assume that there are $\tilde{r}>0$ and $x_{0}, x_{1} \in Z$ such that

$$
\begin{gathered}
J\left(x_{1}\right)=\Phi\left(x_{1}\right)=0, J\left(x_{0}\right)>\tilde{r}, \\
\sup _{x \in J^{-1}((-\infty, \tilde{r}])} \Phi(x)<\tilde{r} \frac{\Phi\left(x_{0}\right)}{J\left(x_{0}\right)} .
\end{gathered}
$$

Then, for each $\beta \in R$ satisfying

$$
\sup _{x \in J^{-1}((-\infty, \tilde{r}])} \Phi(x)<\beta<\tilde{r} \frac{\Phi\left(x_{0}\right)}{J\left(x_{0}\right)},
$$

one has

$\sup _{\eta \geq 0} \inf _{x \in Z}(J(x)+\eta(\beta-\Phi(x)))<\inf _{x \in Z} \sup _{\eta \geq 0}(J(x)+\eta(\beta-\Phi(x)))$.

Lemma 10 (see [14], Theorem B). Let $Z$ be a reflexive Banach space, $J: Z \longrightarrow R$ a continuously Gâteaux differentiable, coercive and sequentially weakly lower semicontinuous functional whose Gâteaux derivative admits a continuous inverse on $Z^{*}$, $\Phi: Z \longrightarrow R$ a continuously Gâteaux differentiable functional whose Gâteaux derivative is compact. Put, for each $\tilde{r}>\inf _{Z} J$,

$$
\begin{aligned}
& \varphi_{1}(\tilde{r})={ }_{x \in J^{-1}}\left(\left(-\infty, r^{\sim}\right)\right) \frac{\sup \frac{\inf }{J^{-1}((-\infty, \tilde{r}))} \omega \Phi-\Phi(x)}{\tilde{r}-J(x)}, \varphi_{2}(\tilde{r}) \\
& =\inf _{x \in J^{-1}((-\infty, \tilde{r}))} \sup _{y \in J^{-1}([\tilde{r},+\infty))} \frac{\Phi(y)-\Phi(x)}{J(y)-J(x)},
\end{aligned}
$$

where $J^{1}((\bar{\infty}, \tilde{r}))^{\omega}$ is the closure of $J^{-1}((-\infty, \tilde{r}))$ in the weak topology, and assume that

(i) there is $\tilde{r} \in R$ such that $\tilde{r}>\inf _{Z} J$ and $\varphi_{1}(\tilde{r})<\varphi_{2}(\tilde{r})$ (ii) $\lim _{\|x\| \rightarrow+\infty}(J(x)-\eta \Phi(x))=+\infty$ for all $\eta \in\left(1 / \varphi_{2}(\tilde{r}), 1 /\right.$ $\left.\varphi_{1}(\tilde{r})\right)$

Then, for each $\eta \in\left(1 / \varphi_{2}(\tilde{r}), 1 / \varphi_{1}(\tilde{r})\right)$, the equation $J^{\prime}(x)$ $-\eta \Phi^{\prime}(x)=0$ has at least three solutions in $Z$.

Next, we recall some definitions and propositions about index theory in $[23,24]$.

Definition 11 (see [24], page 108). For any $B \in L_{s}(X)$, we define

$$
\psi_{a, B}(x, y)=a(x, y)-(B x, y)_{X}, \forall x, y \in Z \text {. }
$$

For any $x, y \in Z$ if $\psi_{a, B}(x, y)=0$, we say that $x$ and $y$ are $\psi_{a, B}$-orthogonal. For any two subspaces $Z_{1}$ and $Z_{2}$ of $Z$ if $\psi_{a, B}(x, y)=0$ for any $x \in Z_{1}, y \in Z_{2}$, we say that $Z_{1}$ and $Z_{2}$ are $\psi_{a, B}$-orthogonal.

Proposition 12 (see [23], Proposition 2.1.1; [24], Proposition 7.2.1). For any $B \in L_{s}(X)$, the space $Z$ has a $\psi_{a, B}$-orthogonal decomposition

$$
Z=Z_{a}^{+}(B) \oplus Z_{a}^{0}(B) \oplus Z_{a}^{-}(B)
$$

such that $\psi_{a, B}$ is positive definite, null, and negative definite on $Z_{a}^{+}(B), Z_{a}^{0}(B)$, and $Z_{a}^{-}(B)$, respectively. Moreover, $Z_{a}^{0}(B)$ and $Z_{a}^{-}(B)$ are finitely dimensional.

Definition 13 (see [23], Definition 2.1.2; [24], Definition 7.2.1). For any $B \in L_{s}(X)$, we define $\nu_{a}(B)=\operatorname{dim} Z_{a}^{0}(B), i_{a}$ $(B)=\operatorname{dim} Z_{a}^{-}(B)$.

Proposition 14 (1) (see [24], Proposition 7.2.2 (i)). For any $B \in L_{s}(X)$, we have

$$
v_{A}(B)=v_{a}(B), i_{A}(B)=i_{a}(B), \operatorname{ker}(A-B)=Z_{a}^{0}(B) .
$$

(2) ([27], Proposition 2.6 (5)). For any $B_{1}, B_{2} \in L_{s}(X)$, if $B_{1} \leq B_{2}$ and $B_{1}<B_{2}$ w.r.t. $\operatorname{ker}\left(A-B_{1}\right)$ and $i_{A}\left(B_{2}\right)=i_{A}\left(B_{1}\right)+$ $v_{A}\left(B_{1}\right)$, then $Z=Z_{a}^{-}\left(B_{1}\right) \oplus Z_{a}^{0}\left(B_{1}\right) \oplus Z_{a}^{0}\left(B_{2}\right) \oplus Z_{a}^{+}\left(B_{2}\right)$, and $\left(-\psi_{a, B_{1}}\left(x_{1}, x_{1}\right)\right)^{1 / 2}+\left(\psi_{a, B_{2}}\left(x_{2}, x_{2}\right)\right)^{1 / 2}$ is an equivalent norm on $Z$ for $x=x_{1}+x_{2}$ with $x_{1} \in Z_{a}^{-}\left(B_{1}\right), x_{2} \in Z_{a}^{+}\left(B_{2}\right)$. In particular, for any $B_{1} \in L_{s}(X)$, then $Z=Z_{a}^{-}\left(B_{1}\right) \oplus Z_{a}^{0}\left(B_{1}\right) \oplus Z_{a}^{+}\left(B_{1}\right)$ and $\left(-\psi_{a, B_{1}}\left(x_{1}, x_{1}\right)\right)^{1 / 2}+\left(\psi_{a, B_{1}}\left(x_{2}, x_{2}\right)\right)^{1 / 2}$ is also an equivalent norm on $Z$ for $x=x_{1}+x_{2}$ with $x_{1} \in Z_{a}^{-}\left(B_{1}\right), x_{2} \in Z_{a}^{+}\left(B_{1}\right)$.

Finally, let us consider two functionals $I$ and $I_{1}$ defined by

$$
\begin{gathered}
I(x)=\frac{1}{2} a(x, x)-\frac{1}{2}\left(B_{0} x, x\right)_{X}-\Phi(x), \\
I_{1}(x)=\frac{1}{2} a(x, x)-\frac{1}{2}\left(B_{0} x, x\right)_{X}+\frac{1}{2}\left(B_{1} x, x\right)_{X}-\eta \Phi(x)-\mu \Psi(x),
\end{gathered}
$$

for every $x \in Z$ and $\eta, \mu \in[0,+\infty)$, respectively. Under assumption $\left(\Phi_{0}\right)$ and $\left(\Psi_{0}\right)$, from Theorem 2 in [28], we can verify 
that $I, I_{1} \in C^{l}(Z, R)$ are weakly lower semicontinuous on $Z$ and $I^{\prime}, I^{\prime}{ }_{1}$ are weakly continuous with

$$
\begin{gathered}
I^{\prime}(x) y=a(x, y)-\left(B_{0} x, y\right)_{X}-\Phi^{\prime}(x) y, \\
I_{1}^{\prime}(x) y=a(x, y)-\left(B_{0} x, y\right)_{X}+\left(B_{1} x, y\right)_{X}-\eta \Phi^{\prime}(x) y-\mu \Psi^{\prime}(x) y,
\end{gathered}
$$

for every $x, y \in Z$ and $\eta, \mu \in[0,+\infty)$.

The following important lemma is an immediate conclusion of Lemma 6 in [26].

Lemma 15. Assume that $\left(\Phi_{0}\right)$ and $\left(\Psi_{0}\right)$ hold. Then, a critical point of $I(x)\left(\right.$ or $\left.I_{1}(x)\right)$ is a solution for problem (3) (or (4)).

\section{Proofs of the Theorems}

In this section, we give the proofs of Theorems $1-4$, respectively. First, with the aid of the critical point reduction method in [4] and the three critical points theorem in [1], we prove Theorem 1. For Theorem 2, we use Theorem 1 in [28] and the three critical points theorem in [1] to give its proof. In the Proof of Theorem 3, our technical approach is based on the three critical points theorem of Ricceri [6]. Finally, we use Proposition 3.1 in [25] and Theorem B in [14] to prove Theorem 4.

Proof of Theorem 1. By assumption $i_{A}\left(B_{0}\right)=0, v_{A}\left(B_{0}\right) \neq 0$, Definition 13, and Proposition 12, we know that $Z=Z_{a}^{0}\left(B_{0}\right)$ $\oplus Z_{a}^{+}\left(B_{0}\right)$. Let $Z_{1}=Z_{a}^{0}\left(B_{0}\right), Z_{2}=Z_{a}^{+}\left(B_{0}\right)$. The functional $\varphi$ is defined by

$$
\varphi\left(x_{2}\right)=\sup _{x_{1} \in Z_{1}} \psi\left(x_{1}+x_{2}\right)=\sup _{x_{1} \in Z_{1}} I\left(x_{1}+x_{2}\right), x_{2} \in Z_{2} .
$$

From the convexity of $\Phi(x)-(1 / 2)\left(B_{3} x, x\right)_{X}$ and assumption $\left(\Phi_{0}\right)$, we can obtain

$$
(\nabla \Phi(x)-\nabla \Phi(y), x-y)_{X} \geq\left(B_{3}(x-y), x-y\right)_{X}, x, y \in Z
$$

Since $B_{3} \in \mathscr{L}_{s}(X)$ is positive definite, there exists $\varepsilon>0$ such that $\left(B_{3} x, x\right)_{X} \geq \varepsilon\|x\|_{X}^{2}$ for all $x \in X$, which shows that

$$
(\nabla \Phi(x)-\nabla \Phi(y), x-y)_{X} \geq \varepsilon\|x-y\|_{X}^{2}, x, y \in Z
$$

Thus, for each fixed $x_{2} \in Z_{2}$ and any $x_{1,1}, x_{1,2} \in Z_{1}$, we obtain

$$
\left(\nabla \Phi\left(x_{1,1}+x_{2}\right)-\nabla \Phi\left(x_{1,2}+x_{2}\right), x_{1,1}-x_{1,2}\right)_{X} \geq \varepsilon\left\|x_{1,1}-x_{1,2}\right\|_{X}^{2}
$$

Since $Z=Z_{1} \oplus Z_{2}=Z_{a}^{0}\left(B_{0}\right) \oplus Z_{a}^{+}\left(B_{0}\right)$, from (39), Defini- tion 11, and Proposition 12, we have

$$
\begin{aligned}
&\left\langle-\psi^{\prime}\left(x_{1,1}+x_{2}\right)-\left(-\psi^{\prime}\left(x_{1,2}+x_{2}\right)\right), x_{1,1}-x_{1,2}\right\rangle \\
&=\left\langle-I^{\prime}\left(x_{1,1}+x_{2}\right)-\left(-I^{\prime}\left(x_{1,2}+x_{2}\right)\right), x_{1,1}-x_{1,2}\right\rangle \\
&=-a\left(x_{1,1}-x_{1,2}, x_{1,1}-x_{1,2}\right)+\left(B_{0}\left(x_{1,1}-x_{1,2}\right), x_{1,1}-x_{1,2}\right)_{X} \\
& \quad+\left(\nabla \Phi\left(x_{1,1}+x_{2}\right)-\nabla \Phi\left(x_{1,2}+x_{2}\right), x_{1,1}-x_{1,2}\right)_{X} \\
&=-\psi_{a, B_{0}}\left(x_{1,1}-x_{1,2}, x_{1,1}-x_{1,2}\right) \\
& \quad+\left(\nabla \Phi\left(x_{1,1}+x_{2}\right)-\nabla \Phi\left(x_{1,2}+x_{2}\right), x_{1,1}-x_{1,2}\right)_{X} \\
& \geq \varepsilon \varepsilon x_{1,1}-x_{1,2} \|_{X}^{2},
\end{aligned}
$$

via $\psi_{a, B_{0}}(x, y)=0$ for all $x, y \in Z_{a}^{0}\left(B_{0}\right)$. Noticing that $Z_{1}=Z_{a}^{0}$ $\left(B_{0}\right)$ is finite-dimensional, we know that there exists $\varepsilon_{0}>0$ such that

$\left\langle-\psi^{\prime}\left(x_{1,1}+x_{2}\right)-\left(-\psi^{\prime}\left(x_{1,2}+x_{2}\right)\right), x_{1,1}-x_{1,2}\right\rangle \geq \varepsilon \varepsilon_{0}\left\|x_{1,1}-x_{1,2}\right\|^{2}$,

which implies that $\psi(x)=I(x)$ satisfies the $\varepsilon \varepsilon_{0}$-monotonicity of Lemma 6. Thus, by Lemma 6, there exists a continuous mapping $\phi: Z_{2} \longrightarrow Z_{1}$ such that $\varphi\left(x_{2}\right)=\psi\left(\phi\left(x_{2}\right)+x_{2}\right)$ for all $x_{2} \in Z_{2}$, where $\varphi: Z_{2} \longrightarrow R$ defined by (19) is continuous differentiable and $\varphi^{\prime}\left(x_{2}\right)=\left.\psi^{\prime}\left(\phi\left(x_{2}\right)+x_{2}\right)\right|_{Z_{2}}$ for all $x_{2} \in Z_{2}$. This means that if $x_{2} \in Z_{2}$ is a critical point of $\varphi, \phi\left(x_{2}\right)+x_{2}$ is a critical point of $\psi$ and $I$.

Again by assumption $\left(\Phi_{1}\right)$, for every $x_{2} \in Z_{2}$, we have

$\left|\Phi\left(x_{2}\right)-\Phi(\theta)\right|=\left|\int_{0}^{1}\left(\nabla \Phi\left(s x_{2}\right), x_{2}\right)_{X} d s\right| \leq \frac{1}{2}\left(B_{2} x_{2}, x_{2}\right)_{X}+c_{0}\left\|x_{2}\right\|$.

Thus, from (19), we have

$$
\begin{aligned}
\varphi\left(x_{2}\right) \geq & \psi\left(x_{2}\right)=I\left(x_{2}\right) \geq \frac{1}{2}\left[a\left(x_{2}, x_{2}\right)-\left(\left(B_{0}+B_{2}\right) x_{2}, x_{2}\right)_{X}\right] \\
& -c_{0}\left\|x_{2}\right\|-\Phi(\theta) \\
= & \frac{1}{2} \psi_{a, B_{0}+B_{2}}\left(x_{2}, x_{2}\right)-c_{0}\left\|x_{2}\right\|-\Phi(\theta)
\end{aligned}
$$

as $x_{1}=\theta$. Since $v_{A}\left(B_{0}+B_{2}\right)=0$ and $i_{A}\left(B_{0}+B_{2}\right)=v_{A}\left(B_{0}\right)$, by (2) of Proposition 14, we can see that $\left(\psi_{a, B_{0}+B_{2}}\left(x_{2}, x_{2}\right)\right)^{1 / 2}$ is an equivalent norm on $Z$ for $x_{2} \in Z_{2}=Z_{a}^{+}\left(B_{0}\right)=Z_{a}^{+}\left(B_{0}+B_{2}\right)$. Hence, there exists $\varepsilon_{1}>0$ such that

$\varphi\left(x_{2}\right) \geq \frac{\varepsilon_{1}}{2}\left\|x_{2}\right\|^{2}-c_{0}\left\|x_{2}\right\|-\Phi(\theta) \geq \frac{\varepsilon_{1}}{2}\left\|x_{2}\right\|^{2}-c_{0}\left\|x_{2}\right\|-\Phi(\theta) \longrightarrow+\infty$,

as $\left\|x_{2}\right\| \longrightarrow+\infty$. Noticing that $I$ is weakly lower semicontinuous on $Z$, then there exists $x_{2,0} \in Z_{2}$ such that $\varphi\left(x_{2,0}\right)=$ 
$\min _{x_{2} \in Z_{2}} \varphi\left(x_{2}\right)$, and hence, $x_{2,0}+\phi\left(x_{2,0}\right)$ is a solution with saddle point character of problem (3) in $Z$.

Moreover, if condition $\left(\Phi_{3}\right)$ holds, then $Z=Z_{a}^{-}\left(B_{01}\right) \oplus$ $Z_{a}^{0}\left(B_{01}\right) \oplus Z_{a}^{0}\left(B_{02}\right) \oplus Z_{a}^{+}\left(B_{02}\right)$ via (2) of Proposition 14. Since $B_{01}>B_{0}$, from Definition 11 , we have $\psi_{a, B_{0}}(x, x)>\psi_{a, B_{01}}(x$, $x)$ for all $x \in Z \backslash\{\theta\}$ and $\psi_{a, B_{0}}(x, x)>0$ for all $x \in Z_{a}^{0}\left(B_{01}\right) \backslash$ $\{\theta\}$ with $Z_{a}^{0}\left(B_{01}\right) \subset Z_{a}^{+}\left(B_{0}\right)$. Let $Z_{2,1}=\left(Z_{a}^{-}\left(B_{01}\right) \oplus Z_{a}^{0}\left(B_{01}\right)\right)$ $\cap Z_{a}^{+}\left(B_{0}\right)$ and $Z_{2,2}$ be the orthogonal complement of $Z_{2,1}$ in $Z_{2}$. We claim that $\phi(\theta)=\theta$. Indeed, $\left(\Phi_{3}\right)$ means that $\Phi(\theta)$ $=0$ and $\nabla \Phi(\theta)=\theta$. By $\left(\Phi_{2}\right)$, (39), and $\phi(\theta) \in Z_{1}=Z_{a}^{0}\left(B_{0}\right)$, we have

$$
\begin{aligned}
0 & =\left\langle\psi^{\prime}(\phi(\theta)),-\phi(\theta)\right\rangle=\left\langle I^{\prime}(\phi(\theta)),-\phi(\theta)\right\rangle=(-\nabla \Phi(\phi(\theta)),-\phi(\theta))_{X} \\
& =(\nabla \Phi(\theta)-\nabla \Phi(\phi(\theta)),-\phi(\theta))_{X} \geq \varepsilon\|\phi(\theta)\|_{X}^{2} \geq 0,
\end{aligned}
$$

which implies that $\phi(\theta)=\theta$. By the continuity of $\phi$, we know that there exists $0<r<\delta / 2$ such that $\left\|\phi\left(x_{2}\right)\right\|<\delta / 2$ as $\left\|x_{2}\right\|$ $\leq r$. Thus, from (12) of $\left(\Phi_{3}\right)$ and Definition 11, we obtain

$$
\begin{aligned}
\varphi\left(x_{2,1}\right)= & \psi\left(x_{2,1}+\phi\left(x_{2,1}\right)\right)=I\left(x_{2,1}+\phi\left(x_{2,1}\right)\right) \\
\leq & \frac{1}{2}\left[a\left(x_{2,1}+\phi\left(x_{2,1}\right), x_{2,1}+\phi\left(x_{2,1}\right)\right)\right. \\
& \left.-\left(B_{01}\left(x_{2,1}+\phi\left(x_{2,1}\right)\right), x_{2,1}+\phi\left(x_{2,1}\right)\right)_{X}\right] \\
= & \frac{1}{2} \psi_{a, B_{01}}\left(x_{2,1}+\phi\left(x_{2,1}\right), x_{2,1}+\phi\left(x_{2,1}\right)\right) \leq 0,
\end{aligned}
$$

for all $x_{2,1} \in Z_{2,1}$ with $\left\|x_{2,1}\right\| \leq r$ via $B_{01}>B_{0}$ and $x_{2,1}+\phi$ $\left(x_{2,1}\right) \in\left(Z_{a}^{-}\left(B_{01}\right) \oplus Z_{a}^{0}\left(B_{01}\right)\right) \cup Z^{0}\left(B_{0}\right)$, and

$$
\begin{gathered}
\varphi\left(x_{2,2}\right) \geq \psi\left(x_{2,2}\right)=I\left(x_{2,2}\right) \\
\geq \frac{1}{2}\left[a\left(x_{2,2}, x_{2,2}\right)-\left(B_{02} x_{2,2}, x_{2,2}\right)_{X}\right]=\frac{1}{2} \psi_{a, B_{02}}\left(x_{2,2}, x_{2,2}\right) \geq 0,
\end{gathered}
$$

for all $x_{2,2} \in Z_{2,2}$ with $\left\|x_{2,2}\right\| \leq r$ via $Z_{2,2}=Z_{a}^{+}\left(B_{01}\right) \cap Z_{a}^{+}\left(B_{0}\right)$ and $Z_{a}^{+}\left(B_{01}\right)=Z_{a}^{+}\left(B_{02}\right) \oplus Z_{a}^{0}\left(B_{02}\right)$.

Since $I$ is weakly lower semicontinuous on $Z$, so is $\varphi$ on $Z_{2}$. From the weakly lower semicontinuity and coerciveness of $\varphi$, we can see that $\varphi$ satisfies (PS) condition and is bounded below on $Z_{2}$.

If inf $\left\{\varphi\left(x_{2}\right): x_{2} \in Z_{2}\right\}=0$, then all $x_{2,1} \in Z_{2,1}$ with $\| x_{2,1}$ $\| \leq r$ are minima of $\varphi$, which shows that $\varphi$ has infinite critical points. If inf $\left\{\varphi\left(x_{2}\right): x_{2} \in z_{2}\right\}<0$, then $\varphi$ has at least two nonzero critical points via Lemma 7. Consequently, problem (3) has at least two nontrivial solutions in $Z$. Further, since $\Phi(\theta)$ $=0$, we know that problem (3) has trivial solution $\theta$. Hence, problem (3) has at least three distinct solutions in $Z$. The proof is complete.

Proof of Theorem 2. By assumption $i_{A}\left(B_{0}\right)=0, v_{A}\left(B_{0}\right) \neq 0$, Definition 13, and Proposition 12, we have $Z=Z_{a}^{0}\left(B_{0}\right) \oplus Z_{a}^{+}$ $\left(B_{0}\right)$. Let $Z_{1}=Z_{a}^{0}\left(B_{0}\right), Z_{2}=Z_{a}^{+}\left(B_{0}\right)$. For $x \in Z$, we have $x=$ $x_{1}+x_{2}$ with $x_{1} \in Z_{1}$ and $x_{2} \in Z_{2}$. It follows from $\left(\Phi_{4}\right)$ that

$$
\begin{aligned}
\left|\Phi(x)-\Phi\left(x_{1}\right)\right|= & \left|\int_{0}^{1}\left(\nabla \Phi\left(x_{1}+s x_{2}\right), x_{2}\right)_{X} d s\right| \\
\leq & c_{1}\left\|x_{2}\right\|_{X_{0}} \int_{0}^{1}\left\|x_{1}+s x_{2}\right\|_{X_{0}}^{\gamma_{0}} d s+c_{2}\left\|x_{2}\right\|_{X_{0}} \\
\leq & c_{1}\left\|x_{2}\right\|_{X_{0}}\left\|x_{1}\right\|_{X_{0}}^{\gamma_{0}}+\frac{c_{1}}{\gamma_{0}+1}\left\|x_{2}\right\|_{X_{0}}^{\gamma_{0}+1}+c_{2}\left\|x_{2}\right\|_{X_{0}} \\
\leq & \frac{c_{1} k_{1} \varepsilon}{2}\left\|x_{2}\right\|^{2}+\frac{c_{1} k_{1}}{2 \varepsilon}\left\|x_{1}\right\|^{2 \gamma_{0}}+\frac{c_{1} k_{1}}{\gamma_{0}+1}\left\|x_{2}\right\|^{\gamma_{0}+1} \\
& +c_{2} k_{1}\left\|x_{2}\right\|,
\end{aligned}
$$

where $\varepsilon$ is any positive real number. Since $x_{2} \in Z_{2}=Z_{a}^{+}\left(B_{0}\right)$, by (2) of Proposition 14, we know that $\left(\psi_{a, B_{0}}\left(x_{2}, x_{2}\right)\right)^{1 / 2}$ is an equivalent norm on $Z$ for $x_{2} \in Z_{2}$. Hence, there exists $\varepsilon_{2}$ $>0$ such that

$$
\left(\psi_{a, B_{0}}\left(x_{2}, x_{2}\right)\right)^{1 / 2} \geq \varepsilon_{2}\left\|x_{2}\right\|
$$

for all $x_{2} \in Z_{2}$, and

$$
\begin{aligned}
I(x)= & \frac{1}{2} \psi_{a, B_{0}}\left(x_{2}, x_{2}\right)-\left[\Phi(x)-\Phi\left(x_{1}\right)\right]-\Phi\left(x_{1}\right) \\
\geq & \frac{\varepsilon_{2}^{2}}{2}\left\|x_{2}\right\|^{2}-\left|\Phi(x)-\Phi\left(x_{1}\right)\right|-\Phi\left(x_{1}\right) \\
\geq & \frac{\varepsilon_{2}^{2}-c_{1} k_{1} \varepsilon}{2}\left\|x_{2}\right\|^{2}-\left\|x_{1}\right\|^{2 \gamma_{0}}\left[\frac{c_{1} k_{1}}{2 \varepsilon}+\frac{\Phi\left(x_{1}\right)}{\left\|x_{1}\right\|^{2 \gamma_{0}}}\right] \\
& -\frac{c_{1} k_{1}}{\gamma_{0}+1}\left\|x_{2}\right\|^{\gamma_{0}+1}-c_{2} k_{1}\left\|x_{2}\right\| .
\end{aligned}
$$

By $c_{3}>c_{1}^{2} k_{1}^{2} / \varepsilon_{2}^{2}>0$, we can choose an $\varepsilon_{0} \in(0,1)$ such that $c_{3}>\left(1+\varepsilon_{0}\right)\left(c_{1}^{2} k_{1}^{2} / \varepsilon_{2}^{2}\right)$. Taking $\varepsilon=\varepsilon_{2}^{2} /\left(c_{1} k_{1}\left(1+\varepsilon_{0}\right)\right)$, by $\left(\Phi_{5}\right)$, we have $\varepsilon_{2}^{2}-c_{1} k_{1} \varepsilon=\left(1-\left(1 /\left(1+\varepsilon_{0}\right)\right)\right) \varepsilon_{2}^{2}>0$ and

$$
\frac{c_{1} k_{1}}{2 \varepsilon}+\frac{\Phi\left(x_{1}\right)}{\left\|x_{1}\right\|^{2 \gamma_{0}}}<\frac{c_{1} k_{1}}{2 \varepsilon}-c_{3}<-\frac{1+\varepsilon_{0}}{2} \cdot \frac{c_{1}^{2} k_{1}^{2}}{\varepsilon_{2}^{2}}<0
$$

for $x_{1} \in Z_{1}=\operatorname{ker}\left(A-B_{0}\right)$. As $\|x\| \longrightarrow+\infty$ if and only if $\| x_{1}$ $\|+\| x_{2} \| \longrightarrow+\infty$, from the above equality and $\left(\Phi_{5}\right)$, we obtain that

$$
I(x) \longrightarrow+\infty \text {, }
$$

as $\|x\| \longrightarrow+\infty$. Noticing that $I$ is weakly lower semicontinuous on $Z$, by Theorem 1 in [28], we know that problem (3) has one solution in $Z$.

Moreover, if condition $\left(\Phi_{3}\right)$ holds, then $Z=Z_{a}^{-}\left(B_{01}\right) \oplus$ $Z_{a}^{0}\left(B_{01}\right) \oplus Z_{a}^{0}\left(B_{02}\right) \oplus Z_{a}^{+}\left(B_{02}\right)$ via (2) of Proposition 14. Let $\bar{Z}_{1}=Z_{a}^{-}\left(B_{01}\right) \oplus Z_{a}^{0}\left(B_{01}\right)$ and $\bar{Z}_{2}=Z_{a}^{0}\left(B_{02}\right) \oplus Z_{a}^{+}\left(B_{02}\right)$. Then, we have $Z=\bar{Z}_{1} \oplus \bar{Z}_{2}$ and $\operatorname{dim} \bar{Z}_{1}<+\infty$. From (12) of $\left(\Phi_{3}\right)$ 
and Definition 11, we obtain

$$
I\left(x_{1}\right) \leq \frac{1}{2}\left[a\left(x_{1}, x_{1}\right)-\left(B_{01} x_{1}, x_{1}\right)_{X}\right]=\frac{1}{2} \psi_{a, B_{01}}\left(x_{1}, x_{1}\right) \leq 0,
$$

for all $x_{1} \in \bar{Z}_{1}$ with $\left\|x_{1}\right\| \leq \delta$, and

$$
I\left(x_{2}\right) \geq \frac{1}{2}\left[a\left(x_{2}, x_{2}\right)-\left(B_{02} x_{2}, x_{2}\right)_{X}\right]=\frac{1}{2} \psi_{a, B_{02}}\left(x_{2}, x_{2}\right) \geq 0,
$$

for all $x_{2} \in \bar{Z}_{2}$ with $\left\|x_{2}\right\| \leq \delta$.

By the weakly lower semicontinuity and coerciveness of $I$, we can see that $I$ satisfies (PS) condition and is bounded below on $Z$. Noticing that $I(x)=-\Phi(x)$ for all $x \in \operatorname{ker}(A-$ $\left.B_{0}\right)$, from (12) of $\left(\Phi_{3}\right)$, we have $I(x)<0$ for all $x \in \operatorname{ker}(A-$ $B_{0}$ ) with $0<\|x\|<\delta$ and $I(\theta)=0$, which implies that $\inf _{Z} I$ $<0$. Thus, $I$ has at least two nonzero critical points via Lemma 7. Thanks to $\theta$ is also a trivial solution of problem (3), we know that problem (3) has at least three distinct solutions in $Z$. The proof is complete.

\section{Proof of Theorem 3. Let}

$$
J(x)=\frac{1}{2} a(x, x)-\frac{1}{2}\left(B_{0} x, x\right)_{X}+\frac{1}{2}\left(B_{1} x, x\right)_{X}=\frac{\|x\|_{B_{1}}^{2}}{2},
$$

for each $x \in Z$. From (33) and (35), we can see that a solution of $J^{\prime}(x)-\eta \Phi^{\prime}(x)-\mu \Psi^{\prime}(x)=0$ must be a critical point of $I_{1}$ $(x)$, which means that problem (4) also has a solution via Lemma 15.

Obviously, $J$ is a continuously Gâteaux differentiable and sequentially weakly lower semicontinuous functional. From the conditions $\left(\Phi_{0}\right)$ and $\left(\Psi_{0}\right)$, we know that $\Phi$ and $\Psi$ are continuously Gâteaux differentiable functional whose Gâteaux derivative is compact. Moreover, $J$ is bounded on each bounded subset of $Z$ via (8).

Next, we prove that $J$ admits a continuous inverse on $Z$. In fact, from (8) and (35), we have

$$
\left\langle J^{\prime}(x)-J^{\prime}(y), x-y\right\rangle=\|x-y\|_{B_{1}}^{2} \geq \min \{1, \underline{\lambda}\}\|x-y\|^{2},
$$

for all $x, y \in Z$, which shows that $J^{\prime}$ is uniformly monotone on $Z$. Moreover, using standard arguments ensure that $J^{\prime}$ also turns out to be coercive and hemicontinuous in $Z$. Applying Theorem 26.A of [29], we know that $J$ admits a continuous inverse on $Z$.

Thanks to $\left(\Phi_{7}\right)$ and (8), for each $\eta>0$, we have

$$
\lim _{\|x\| \rightarrow+\infty}(J(x)-\eta \Phi(x)) \geq \lim _{\|x\| \rightarrow+\infty}\left(\frac{\|x\|_{B_{1}}^{2}}{2}-\eta\left(c_{3}\|x\|_{X_{0}}^{v}+c_{4}\right)\right)=+\infty,
$$

via $\gamma \in(0,2)$ and $\|x\|_{X_{0}} \leq k_{1}\|x\|$. This implies that the assumption (22) of Lemma 8 holds.
For $x_{0} \in \operatorname{ker}\left(A-B_{0}\right)$ in $\left(\Phi_{8}\right)$, by the definition of $\|\cdot\|_{B_{1}}$, we have

$$
\sqrt{\bar{\lambda}}\left\|x_{0}\right\|_{X} \geq\left\|x_{0}\right\|_{B_{1}}=\left(\left(B_{1} x_{0}, x_{0}\right)_{X}\right)^{1 / 2} \geq \sqrt{\lambda}\left\|x_{0}\right\|_{X} .
$$

From $\tilde{c}<\tilde{k}\left\|x_{0}\right\|_{X} \sqrt{\underline{\lambda}}$, we see that

$$
\left\|x_{0}\right\|_{B_{1}}>\frac{\tilde{c}}{\tilde{k}} .
$$

Hence, thanks to (15) of $\left(\Phi_{8}\right)$ and (58), we have

$$
\sup _{x \in E} \Phi(x)<\frac{\tilde{c}^{2}}{\bar{\lambda} \tilde{k}^{2}\left\|x_{0}\right\|_{X}^{2}} \Phi\left(x_{0}\right) \leq \frac{\tilde{c}^{2}}{\tilde{k}^{2}\left\|x_{0}\right\|_{B_{1}}^{2}} \Phi\left(x_{0}\right) .
$$

Now, taking $\tilde{r}=(1 / 2)(\tilde{c} / \tilde{k})^{2}$. By (59), we have

$$
J\left(x_{0}\right)=\frac{\left\|x_{0}\right\|_{B_{1}}^{2}}{2}>\tilde{r}>0=J(\theta) .
$$

From (9) and (10), we obtain $\|x\|_{X_{0}} \leq \tilde{k}\|x\|_{B_{1}}$ for all $x \in Z$. Hence, for each $x \in Z$ such that

$$
J(x)=\frac{\|x\|_{B_{1}}^{2}}{2} \leq \tilde{r},
$$

we have $(1 / 2)\left(\|x\|_{X_{0}} / k\right)^{2} \leq \tilde{r}=(1 / 2)(\tilde{c} / k)^{2}$, which implies that

$$
\|x\|_{X_{0}} \leq \tilde{c} .
$$

It follows from (60) and (63) that

$$
\sup _{x \in J^{-1}((-\infty, \tilde{r}))} \Phi(x) \leq \sup _{x \in E} \Phi(x)<\frac{\tilde{c}^{2}}{\tilde{k}^{2}\left\|x_{0}\right\|_{B_{1}}^{2}} \Phi\left(x_{0}\right)=\tilde{r} \frac{\Phi\left(x_{0}\right)}{J\left(x_{0}\right)} .
$$

Put $\beta$ satisfying

$$
\sup _{x \in J^{-1}((-\infty, \tilde{r}])} \Phi(x)<\beta<\tilde{r} \frac{\Phi\left(x_{0}\right)}{J\left(x_{0}\right)},
$$

and $x_{1}=\theta$, by (61), (64), and Proposition 9, we have

$$
\sup _{\eta \geq 0} \inf _{x \in Z}(J(x)+\eta(\beta-\Phi(x)))<\inf _{x \in Z} \sup _{\eta \geq 0}(J(x)+\eta(\beta-\Phi(x))),
$$

which implies that the assumption (23) of Lemma 8 holds as $\tilde{I}=[0,+\infty)$.

Finally, set $\tilde{I}=[0,+\infty)$, we can see that all the assumptions of Lemma 8 are satisfied. Hence, our conclusion follows from Lemma 8.

Proof of Theorem 4. In order to use Lemma 10 to prove Theorem 4 , we consider the functional $I_{1}$ defined by (33) as $\mu=0$. From the Proof of Theorem 3, it is not difficult to see that we only need to verify the validity of (i) in Lemma 10. 
From the Proof of Theorem 3, we know that $J(\theta)=$ $\Phi(\theta)=0, \tilde{r}=(1 / 2)(\tilde{c} / k)^{2}$, and

$$
J\left(x_{0}\right)=\frac{\left\|x_{0}\right\|_{B_{1}}^{2}}{2}>\tilde{r}>0=\inf _{Z} J .
$$

This shows that $\theta \in J^{-1}((-\infty, \tilde{r}))$ and $x_{0} \in J^{-1}([\tilde{r},+\infty))$. From $(63)$ and $\left(\Phi_{9}\right)$, we have

$$
\sup _{x \in J^{-1}((-\infty, \tilde{r}])} \Phi(x) \leq \sup _{x \in E} \Phi(x)<\frac{\tilde{c}^{2}}{\tilde{c}^{2}+\bar{\lambda} \tilde{k}^{2}\left\|x_{0}\right\|_{X}^{2}} \Phi\left(x_{0}\right)<\Phi\left(x_{0}\right),
$$

which implies that

$$
\Phi\left(x_{0}\right)-\Phi(x)>0
$$

for each $x \in J^{-1}((-\infty, \tilde{r}]) \subset Z$. Consequently, it follows from $\left(\Phi_{9}\right),(58)$ and $(69)$ that

$$
\begin{aligned}
\varphi_{1}(\tilde{r}) & =\inf _{x \in J^{-1}((-\infty, \tilde{r}))} \frac{\sup ^{-1}((-\infty, \tilde{r}])}{\tilde{r}-J(x)} \leq \frac{\sup _{(x)} \Phi-\Phi(\theta)}{J^{-1}((-\infty, \tilde{r}])} \\
& =\frac{\sup _{J^{-1}((-\infty, \tilde{r}])} \Phi}{\tilde{r}} \leq \frac{\sup _{x \in E} \Phi(x)}{\tilde{r}}=\frac{2 \tilde{k}^{2} \sup _{x \in E} \Phi(x)}{\tilde{c}^{2}} \\
& <\frac{2 \tilde{k}^{2}}{\tilde{c}^{2}+\bar{\lambda} \tilde{k}^{2}\left\|x_{0}\right\|_{X}^{2}} \Phi\left(x_{0}\right),
\end{aligned}
$$

$$
\begin{aligned}
& \varphi_{2}(\tilde{r}) \geq \inf _{x \in J^{-1}((-\infty, \tilde{r}))} \frac{\Phi\left(x_{0}\right)-\Phi(x)}{J\left(x_{0}\right)-J(x)} \geq \frac{\inf _{x \in J^{-1}((-\infty, \tilde{r}))} \Phi\left(x_{0}\right)-\Phi(x)}{J\left(x_{0}\right)} \\
& \geq \frac{2}{\left\|x_{0}\right\|_{B_{1}}^{2}}\left(\Phi\left(x_{0}\right)-\sup _{x \in J^{-1}((-\infty, \tilde{r}])} \Phi(x)\right) \\
& \geq \frac{2}{\bar{\lambda}\left\|x_{0}\right\|_{X}^{2}}\left(\Phi\left(x_{0}\right)-\sup _{x \in E} \Phi(x)\right) \\
& >\frac{2}{\bar{\lambda}\left\|x_{0}\right\|_{X}^{2}}\left(\Phi\left(x_{0}\right)-\frac{\tilde{c}^{2}}{\tilde{c}^{2}+\bar{\lambda} \tilde{k}^{2}\left\|x_{0}\right\|_{X}^{2}} \Phi\left(x_{0}\right)\right) \\
& \geq \frac{2 \tilde{k}^{2}}{\tilde{c}^{2}+\bar{\lambda} \tilde{k}^{2}\left\|x_{0}\right\|_{X}^{2}} \Phi\left(x_{0}\right) \text {. }
\end{aligned}
$$

From (70) and (71), we obtain

$$
\begin{gathered}
\varphi_{2}(\tilde{r})>\varphi_{1}(\tilde{r}), \\
\left.\frac{\bar{\lambda}\left\|x_{0}\right\|_{X}^{2}}{2\left(\Phi\left(x_{0}\right)-\sup _{x \in E} \Phi(x)\right)}, \frac{\tilde{c}^{2}}{2 \tilde{k}^{2} \sup _{x \in E} \Phi(x)}\right) \subset\left(\frac{1}{\varphi_{2}(\tilde{r})}, \frac{1}{\varphi_{1}(\tilde{r})}\right) .
\end{gathered}
$$

Therefore, (i) of Lemma 10 is satisfied. The proof is complete.

\section{Applications to Second-Order Hamiltonian Systems and Elliptic Partial Differential Equations}

In this section, as three examples of applications of the main results, we investigate second-order Hamiltonian systems satisfying generalized periodic boundary value conditions, second-order Hamiltonian systems satisfying SturmLiouville boundary value conditions, and elliptic partial differential equations satisfying Dirichlet boundary value conditions. For systematic researches of Hamiltonian systems, we refer to the excellent books [28, 30, 31]. Meanwhile, for researches of elliptic partial differential equations, we refer to [32-34].

4.1. Second-Order Hamiltonian Systems Satisfying Generalized Periodic Boundary Value Conditions. As a first example, we discuss the solvability of the following two generalized periodic boundary value problems

$$
\begin{gathered}
\left\{\begin{array}{l}
-\ddot{x}-\bar{B}_{0}(t) x=\nabla_{x} V(t, x), \\
x(1)=M x(0), \dot{x}(1)=N \dot{x}(0),
\end{array} \quad \text { a.e.t } \in[0,1],\right. \\
\left\{\begin{array}{l}
-\ddot{x}-\bar{B}_{0}(t) x+\bar{B}_{1}(t) x=\eta \nabla_{x} V(t, x)+\mu \nabla_{x} F(t, x), \quad \text { a.e.t } \in[0,1], \\
x(1)=M x(0), \dot{x}(1)=N \dot{x}(0),
\end{array}\right.
\end{gathered}
$$

where $\eta, \mu \in[0,+\infty), \bar{B}_{0}(t), \bar{B}_{1}(t) \in L^{\infty}\left([0,1], L_{s}\left(R^{n}\right)\right)=\{B(t)$ $\left.=\left(b_{j k}\right)_{n \times n} \mid b_{j k}(t)=b_{k j}(t), t \in[0,1], b_{j k}(t) \in L^{\infty}([0,1])\right\}, M, N$ $\in G L(n)=\left\{A=\left(a_{j k}\right)_{n \times n} \mid a_{j k} \in \operatorname{Randdet}(A) \neq 0\right\}$, and $M N^{T}$ $=I_{n}$, where $I_{n}$ is the unit matrix of order $n$, and $\nabla_{x} V(t, x)$ and $\nabla_{x} F(t, x)$ denote the gradient of $V(t, x)$ and $F(t, x)$ w.r.t. $x \in R^{n}$, respectively.

Let $X=L^{2}\left([0,1], R^{n}\right)$. We define $A_{1}: D\left(A_{1}\right) \longrightarrow X$ by $\left(A_{1} x\right)(t)=-\ddot{x}(t) \quad$ where $\quad D\left(A_{1}\right)=\left\{x \in H^{2}\left([0,1], R^{n}\right) \mid x\right.$ satisfies $x(1)=M x(0), \dot{x}(1)=N \dot{x}(0)\}$. Set $\left(B_{i} x\right)(t)=\bar{B}_{i}(t) x$ $(t)(i=0,1)$. Then, $D\left(B_{i}\right)=X$. From Corollary 1.21 in [26], we see that $A_{1}$ is self-adjoint and $\sigma\left(A_{1}\right)=\sigma_{d}\left(A_{1}\right)$ $C[0,+\infty)$. Define $i_{M}\left(\bar{B}_{0}\right)=i_{A_{1}}\left(B_{0}\right), v_{M}\left(\bar{B}_{0}\right)=v_{A_{1}}\left(B_{0}\right)$, that is, $v_{M}\left(\bar{B}_{0}\right)$ is the dimension of the solution subspace of (73) as $V(t, x) \equiv 0$ and $i_{M}\left(\bar{B}_{0}\right)=\sum_{\lambda<0} v_{M}\left(\bar{B}_{0}+\lambda I_{n}\right)$.

Remark 16 (see [23], Example 2.4.3; [24], Remark 7.1.3). Let $\alpha_{1} \leq \alpha_{2} \leq \cdots \leq \alpha_{n}$ be the eigenvalues of a constant $n \times n$ symmetric matrix $B$. For $\zeta \in R \backslash\{0\}$ with $\xi_{0}=\arccos 2 /\left(\zeta^{-1}+\zeta\right)$, we have

$$
\begin{aligned}
i_{\zeta I_{n}}(B)= & \sum_{k=1}^{n} \#\left\{j \in N:\left(2 j \pi+\xi_{0}\right)^{2}<\alpha_{k}\right\} \\
& +\sum_{k=1}^{n} \#\left\{j \in N:\left(2 \pi-\xi_{0}+2 j \pi\right)^{2}<\alpha_{k}\right\},
\end{aligned}
$$




$$
\begin{aligned}
\nu_{\zeta I_{n}}(B)= & \sum_{k=1}^{n} \#\left\{j \in N:\left(2 j \pi+\xi_{0}\right)^{2}=\alpha_{k}\right\} \\
& +\sum_{k=1}^{n} \#\left\{j \in N:\left(2 \pi-\xi_{0}+2 j \pi\right)^{2}=\alpha_{k}\right\},
\end{aligned}
$$

where ${ }^{\#} S$ denotes the number of elements in a set $S$. In particular, formulae (75) and (76) when $\zeta=1$ were given first by Mawhin and Willem in [28].

Now, we assume that $\bar{B}_{1}(t)$ is positive definite for all $x$ $\in R^{n}$, that is, there exists a positive constant $\underline{\lambda}$ such that $\left(\bar{B}_{1}\right.$ $(t) x, x) \geq \underline{\lambda}|x|^{2}$ for all $x \in R^{n}$, and $V, F:[0,1] \times R^{n} \longrightarrow R$ satisfy the following condition:

$\left(H_{0}\right) V(t, x)$ and $F(t, x)$ are measurable in $t$ for every $x$ $\in R^{n}$ and continuously differentiable in $x$ for a.e. $t \in[0,1]$.

Further, assume that $i_{M}\left(\bar{B}_{0}\right)=0, v_{M}\left(\bar{B}_{0}\right) \neq 0$. Meanwhile, set $Z_{1}=\left\{x \in H^{1}\left([0,1], R^{n}\right) \mid x(1)=M x(0)\right\}$. By Corollary 1.21 in [26] again, we know that $Z_{1}=D\left(\left|A_{1}\right|^{1 / 2}\right)$ and the norm

$$
\|x\|_{Z_{1}}=\left(\int_{0}^{1}\left[|\dot{x}(t)|^{2}+|x(t)|^{2}\right] d t\right)^{1 / 2}
$$

for each $x \in Z_{1}$. Noticing that $i_{M}\left(\bar{B}_{0}\right)=0, v_{M}\left(\bar{B}_{0}\right) \neq 0$, we can define another equivalent norm

$$
\|x\|=\left(\int_{0}^{1}\left[|\dot{x}(t)|^{2}-\left(\bar{B}_{0}(t) x(t), x(t)\right)+|x(t)|^{2}\right] d t\right)^{1 / 2}
$$

for each $x \in Z_{1}$.

Let

$$
\begin{aligned}
\|x\|_{\bar{B}_{1}}= & \left(\int _ { 0 } ^ { 1 } \left[|\dot{x}(t)|^{2}-\left(\bar{B}_{0}(t) x(t), x(t)\right)\right.\right. \\
& \left.\left.+\left(\bar{B}_{1}(t) x(t), x(t)\right)\right] d t\right)^{1 / 2}, \quad \forall x \in Z_{1} .
\end{aligned}
$$

Since $\bar{B}_{1}(t) \in L^{\infty}\left([0,1], L_{s}\left(R^{n}\right)\right)$ is positive definite, there exist $\bar{\lambda}>\underline{\lambda}>0$ such that $\bar{\lambda}\|x\|_{L^{2}}^{2} \geq \int_{0}^{1}\left(\bar{B}_{1}(t) x(t), x(t)\right) d t \geq \underline{\lambda}$ $\|x\|_{L^{2}}^{2}$ for all $x \in X$. So, we have

$$
\min \{1, \underline{\lambda}\}\|x\|^{2} \leq\|x\|_{\bar{B}_{1}}^{2} \leq \max \{1, \bar{\lambda}\}\|x\|^{2} .
$$

Observe that the embedding $Z_{1}{ }^{\circ} L^{2}=X$ is compact. Let $\| \cdot$ $\|_{\infty}$ be the norm of $L^{\infty}\left([0,1], R^{n}\right)$. Then, there is a constant $\delta_{0}>0$ such that

$$
|x| \leq\|x\|_{\infty} \leq \delta_{0}\|x\|
$$

for any $x \in Z_{1}$. Let

$$
k_{2}=\sup _{x \in Z_{1} \backslash\{\theta\}} \frac{\|x\|_{\infty}}{\|x\|_{\bar{B}_{1}}} .
$$

From (80) and (81), we have

$$
0<k_{2} \leq \tilde{k}=\delta_{0}(\min \{1, \underline{\lambda}\})^{-1 / 2}
$$

For any $\bar{B}_{1}(t), \bar{B}_{2}(t) \in L^{\infty}\left([0,1], L_{s}\left(R^{n}\right)\right)$, we write $\bar{B}_{1} \leq \bar{B}_{2}$ if $\bar{B}_{1}(t) \leq \bar{B}_{2}(t)$ for a.e. $t \in[0,1]$ and define $\bar{B}_{1}<\bar{B}_{2}$ if $\bar{B}_{1}(t)$ $<\bar{B}_{2}(t)$ a.e. $t \in[0,1]$. From Theorems $1-4$, we have the following four results hold.

Theorem 17. Assume that $V(t, x)$ satisfies $\left(H_{0}\right)$ and

$\left(H_{1}\right)$ 5there exist $g \in L^{1}\left([0,1], R^{+}\right)$and $\bar{B}_{2} \in L^{\infty}([0,1]$, $\left.L_{s}\left(R^{n}\right)\right)$ with $\bar{B}_{2} \geq 0, v_{M}\left(\bar{B}_{0}+\bar{B}_{2}\right)=0$ and $i_{M}\left(\bar{B}_{0}+\bar{B}_{2}\right)=v_{M}$ $\left(\bar{B}_{0}\right)$ such that

$$
\left|\nabla_{x} V(t, x), x\right| \leq\left(\bar{B}_{2}(t) x, x\right)+g(t)|x|,
$$

for all $x \in R^{n}$ and a.e. $t \in[0,1]$

$\left(H_{2}\right)$ there exists a positive definite $\bar{B}_{3} \in L^{\infty}\left([0,1], L_{s}\left(R^{n}\right)\right)$ such that $V(t, x)-1 / 2\left(\bar{B}_{3}(t) x, x\right)$ is convex in $x$ for a.e. $t \in[0,1]$

Then, problem (73) has at least one solution with saddle character in $Z_{1}$.

Assume in addition that

$\left(H_{3}\right)$ there exist $\delta_{1}>0, \bar{B}_{01}, \bar{B}_{02} \in L^{\infty}\left([0,1], L_{s}\left(R^{n}\right)\right)$ such that $\bar{B}_{02}>\bar{B}_{01}>\bar{B}_{0}$ and $v_{M}\left(\bar{B}_{0 i}\right) \neq 0(i=1,2), i_{M}\left(\bar{B}_{02}\right)=i_{M}$ $\left(\bar{B}_{01}\right)+v_{M}\left(\bar{B}_{01}\right)$, and for all $|x| \leq \delta_{1}$

$$
\frac{1}{2}\left(\left(\bar{B}_{01}(t)-\bar{B}_{0}(t)\right) x, x\right) \leq V(t, x) \leq \frac{1}{2}\left(\left(\bar{B}_{02}(t)-\bar{B}_{0}(t)\right) x, x\right),
$$

for a.e. $t \in[0,1]$. Then, problem (73) has at least three distinct solutions in $Z_{1}$.

Proof. Set

$$
\Phi(x)=\int_{0}^{1} V(t, x) d t, \forall x \in Z_{1}
$$

Then, $\Phi \in C^{1}\left(Z_{1}, R\right)$ is weakly continuous with compact derivative, and for every $x \in Z_{1}$,

$$
\Phi^{\prime}(x) y=\int_{0}^{1}\left(\nabla_{x} V(t, x), y\right) d t, \forall y \in Z_{1}
$$

because of $\left(\mathrm{H}_{0}\right)$ and (84). Hence, $\left(\Phi_{0}\right)$ holds.

Thanks to (81) and (84) of $\left(H_{1}\right)$, we have

$$
\begin{aligned}
\left|(\nabla \Phi(x), x)_{L^{2}}\right| & \leq \int_{0}^{1}\left(\bar{B}_{2}(t) x(t), x(t)\right) d t+\int_{0}^{1} g(t)|x(t)| d t \\
& \leq\left(B_{2} x, x\right)_{L^{2}}+\|x\|_{\infty} \int_{0}^{1} g(t) d t \\
& \leq\left(B_{2} x, x\right)_{L^{2}}+c_{0}\|x\|,
\end{aligned}
$$


for all $x \in L^{2}$, where $c_{0}=\delta_{0} \int_{0}^{1} g(t) d t$. This shows that $\left(\Phi_{1}\right)$ holds.

Since $\bar{B}_{3}(t)$ is positive definite, that is, there exists a positive constant $\varepsilon_{0}$ such that $\left(\bar{B}_{3}(t) x, x\right) \geq \varepsilon_{0}|x|^{2}$ for all $x \in$ $R^{n}$, which implies that $\left(B_{3} x, x\right)_{L^{2}} \geq \varepsilon_{0}\|x\|_{L^{2}}^{2}$ for all $x \in L^{2}$. Thus, $\left(\Phi_{2}\right)$ follows from $\left(H_{2}\right)$.

Clearly, $\bar{B}_{02}>\bar{B}_{01}>\bar{B}_{0} \Longrightarrow B_{02}>B_{01}>B_{0}$. Noticing that $|x| \leq\|x\|_{\infty} \leq \delta_{0}\|x\|$ for any $x \in Z_{1}$, we have $|x| \leq \delta_{1}$ as $\|x\|$ $\leq \delta_{1} / \delta_{0}=\delta$. So (12) follows from (85), which shows that $\left(\Phi_{3}\right)$ holds. Now, we can apply Theorem 1 to obtain Theorem 17.

Remark 18. In particular, set $\bar{B}_{0}(t) \equiv 0, \bar{B}_{2}(t)=f(t) I_{n}, \bar{B}_{3}(t)$ $=h(t) I_{n}, \bar{B}_{01}(t)=m^{2}(2 \pi)^{2} I_{n}, \bar{B}_{02}(t)=(m+1)^{2}(2 \pi)^{2} I_{n}, m \epsilon$ $\{1,2, \cdots\}$ and $M=I_{n}$, where $f(t), h(t) \in L^{1}\left([0,1], R^{+}\right)$with 0 $<f(t)<4 \pi^{2}$ and $\inf _{t \in[0,1]} h(t)>0$. Then, $Z_{1}=\left\{x \in H^{1}([0,1]\right.$, $\left.\left.R^{n}\right) \mid x(1)=x(0)\right\}, \sigma\left(A_{1}\right)=\left\{(2 m \pi)^{2} \mid m \in N\right\}$, and $\operatorname{ker}\left(A_{1}-\right.$ $\left.\bar{B}_{0}\right)=R^{n}$. Hence, the following problem

$$
-\ddot{x}(t)=\nabla_{x} V(t, x(t)), x(0)-x(1)=\dot{x}(0)-\dot{x}(1)=0
$$

has a similar conclusion of Theorem 17. In addition, for the interval $[0, T]$ considered in second-order Hamiltonian systems satisfying periodic boundary value conditions. As $T=$ 1, the conclusion of Theorem 2.2 in [3] holds if we replace $0<f(t)<4 \pi^{2}$ and $\inf _{t \in[0,1]} h(t)>0$ with $0<\int_{0}^{1} f(t) d t<12$ and $\int_{0}^{1} h(t) d t>0$, respectively. Clearly, inf $\operatorname{te[0,1]}_{(t)>0 \Longrightarrow} h(t$ $\int_{0}^{1} h(t) d t>0$. But for $f \in L^{1}\left([0,1], R^{+}\right)$, we can see that $\int_{0}^{1}$ $f(t) d t<12 \Longrightarrow 0<f(t)<4 \pi^{2}$ and $0<f(t)<4 \pi^{2} \Longrightarrow \int_{0}^{1} f(t) d$ $t<12$. Indeed, if

$$
f(t)= \begin{cases}8 \pi^{2}, & x \in\left[0, \frac{1}{4 \pi^{2}}\right], \\ 1-\frac{1}{4 \pi^{2}}, & x \in\left(\frac{1}{4 \pi^{2}}, 1\right]\end{cases}
$$

then $\int_{0}^{1} f(t) d t=3$ and $f(t)>4 \pi^{2}$ for $x \in\left[0,1 / 4 \pi^{2}\right]$; if 12 $<f(t)<4 \pi^{2}$, then $\int_{0}^{1} f(t) d t>12$. So Theorem 17 is a new result and in a sense a development of Theorem 2.2 in [3].

Next, we give some examples about the problem (89).

Example 1. Let $f(t)=2 \pi^{2}\left(1+\kappa_{0} \sin ^{2} t\right), g(t)=4 \pi^{2}, h(t)=2$ $\pi^{2}, \bar{B}_{01}(t)=(2 \pi)^{2} I_{n}, \bar{B}_{02}(t)=4(2 \pi)^{2} I_{n}$. For each $\kappa_{0} \in(0,1 /$ $\left.\sin ^{2} 1\right)$, by the convexity of

$$
Y(s)= \begin{cases}2 \pi^{2} k s-\pi^{2}, & s \geq 1, \\ \pi^{2} k s^{2}, & 0 \leq s \leq 1,\end{cases}
$$

where $k>0$, we can verify that

$$
V(t, x)= \begin{cases}\pi^{2}\left(1+\kappa_{0} \sin ^{2} t\right)|x|^{2}+2 \pi^{2}\left(1+\kappa_{0} \sin ^{2} t\right)|x|-\pi^{2}\left(1+k_{0} \sin ^{2} t\right), & |x| \geq 1, \\ 2 \pi^{2}\left(1+\kappa_{0} \sin ^{2} t\right)|x|^{2}, & |x| \leq 1\end{cases}
$$

satisfies the conditions of Theorem 17. So problem (89) has at least three distinct solutions. Noticing that $\int_{0}^{1} f(t) d t>2 \pi^{2}$ $>12$, we know that Example 1 does not satisfy the conditions of Theorem 2.2 in [3].

Theorem 19. Assume that $V(t, x)$ satisfies $\left(H_{0}\right)$ and that

$\left(H_{4}\right)$ there exist $f, g \in L^{1}\left([0,1], R^{+}\right)$and $\gamma_{0} \in(0,1)$ such

$$
\left|\nabla_{x} V(t, x)\right| \leq f(t)|x|^{\gamma_{0}}+g(t)
$$

for all $x \in R^{n}$ and a.e. $t \in[0,1]$

$\left(H_{5}\right)$ there exists $c_{3}>\left(\delta_{0}^{2} / 2 \varepsilon_{2}^{2}\right)\left(\int_{0}^{1} f(t) d t\right)^{2}>0$ such that

$$
\limsup _{\|x\| \rightarrow+\infty}\|x\|^{-2 \gamma_{0}} \int_{0}^{1} V(t, x) d t<-c_{3}
$$

for all $x \in \operatorname{ker}\left(A_{1}-\bar{B}_{0}\right)$, where the constants $\delta_{0}$ and $\varepsilon_{2}$ are given by (81) and (49), respectively.
Then, problem (73) has at least one solution in $Z_{1}$. Further, assume that $V(t, x)$ satisfies $\left(H_{3}\right)$. Then, problem (73) has at least three distinct solutions in $Z_{1}$.

Proof. Similar to the proof of Theorem 17, we need only to show that $\left(\Phi_{4}\right)$ follows from $\left(H_{4}\right)$. By $\left(H_{4}\right)$, we have

$$
\begin{aligned}
\left|(\nabla \Phi(x), y)_{L^{2}}\right| & \leq \int_{0}^{1} f(t)|x(t)|^{\gamma_{0}} \cdot|y(t)| d t+\int_{0}^{1} g(t)|y(t)| d t \\
& \leq c_{1}\|x\|_{\infty}^{\gamma_{0}} \cdot\|y\|_{\infty}+c_{2}\|x\|_{\infty},
\end{aligned}
$$

for all $x, y \in L^{2}$, where $c_{1}=\int_{0}^{1} f(t) d t, c_{2}=\int_{0}^{1} g(t) d t$. This shows that $\left(\Phi_{4}\right)$ holds. Hence, we can apply Theorem 2 to obtain Theorem 19.

Remark 20. In particular, set $\bar{B}_{0}(t) \equiv 0, \bar{B}_{01}(t)=m^{2}(2 \pi)^{2} I_{n}$, $\bar{B}_{02}(t)=(m+1)^{2}(2 \pi)^{2} I_{n}, m \in\{1,2, \cdots\}$ and $M=I_{n}$. Then, the following problem (89) has a similar conclusion of Theorem 19. For the interval $[0, T]$ considered in second-order Hamiltonian systems satisfying periodic boundary value 
conditions, as $T=1$, the conclusion of Theorem 4 in [2] holds if we replace $\left(H_{5}\right)$ with

$$
\lim _{|x| \rightarrow+\infty}|x|^{-2 \gamma_{0}} \int_{0}^{1} V(t, x) d t=-\infty .
$$

Clearly, our result generalizes Theorem 4 in [2].

In addition, as $T=1$, in Theorem 7 of [1], assume that the smooth periodic function $V(t, x)$ satisfies $\left(\mathrm{H}_{3}\right)$,

$$
\left(H_{5}^{\prime}\right) V(t, x) \longrightarrow-\infty \text { as }|x| \longrightarrow+\infty \text { uniformly in } t
$$

and for some $x_{0} \in R^{n}$,

$$
\int_{0}^{1} V\left(t, x_{0}\right) d t>0
$$

Then, problem (89) has at least three distinct solutions. Clearly, condition $\left(\mathrm{H}_{4}\right)$ is stronger than condition of Theorem 7 in [1], and condition $\left(H_{5}\right)$ is weaker than condition $\left(H^{\prime}{ }_{5}\right)$. Moreover, we can see that the below Example 2 satisfies the conditions of Theorem 19 but does not satisfy Theorem 7 in [1]. So Theorem 19 is also a generalization of Theorem 7 in [1] in a sense. Next, we give an example about problem (89).

\section{Example 2. Let}

$$
V(t, x)= \begin{cases}(\sin 2 \pi t)|x|^{\frac{7}{4}}+\left(\frac{1}{5}-t\right)|x|^{3 / 2}+\left[4 \pi^{2} \sin ^{2} \pi t-\frac{7}{4} \sin 2 \pi t+8 \pi^{2},\right. & \\ \left.+\frac{3}{2}\left(t-\frac{1}{5}\right)\right]|x|-2 \pi^{2} \sin ^{2} \pi t+\frac{3}{4} \sin 2 \pi t-4 \pi^{2}-\frac{1}{2}\left(t-\frac{1}{5}\right), & |x| \geq 1, \\ 2 \pi^{2}\left(2+\sin ^{2} \pi t\right)|x|^{2}, & |x| \leq 1\end{cases}
$$

for all $x \in R^{n}$ and $t \in[0,1]$. It is easy to see that

$$
\nabla_{x} V(t, x)= \begin{cases}\frac{7}{4}(\sin 2 \pi t) \frac{x}{|x|^{1 / 4}}+\frac{3}{2}\left(\frac{1}{5}-t\right) \frac{x}{|x|^{1 / 2}}+\left[4 \pi^{2} \sin ^{2} \pi t-\frac{7}{4} \sin 2 \pi t,\right. & \\ \left.+8 \pi^{2}+\frac{3}{2}\left(t-\frac{1}{5}\right)\right] \frac{x}{|x|}, & |x| \geq 1 \\ 4 \pi^{2}\left(2+\sin ^{2} \pi t\right) x, & |x| \leq 1\end{cases}
$$

for all $x \in R^{n}$ and a.e. $t \in[0,1]$. Note that for $\varepsilon>0$, we have

$$
\frac{6}{5}|x|^{1 / 2} \leq \frac{7}{4} \varepsilon|x|^{3 / 4}
$$

as $x \in R^{n}$ and $|x| \geq 1 / \varepsilon^{4}$, which implies that $(6 / 5)|x|^{1 / 2} \leq(7 / 4$ )$\varepsilon|x|^{3 / 4}+\left(6 / 5 \varepsilon^{2}\right)$ and

$$
\left|\nabla_{x} V(t, x)\right| \leq \frac{7}{4}(|\sin 2 \pi t|+\varepsilon)|x|^{3 / 4}+\frac{6}{5 \varepsilon^{2}}+13 \pi^{2}
$$

for all $x \in R^{n}$ and a.e. $t \in[0,1]$. The above shows $\left(H_{4}\right)$ holds with $\gamma_{0}=3 / 4, f(t)=(7 / 4)(|\sin 2 \pi t|+\varepsilon), g(t)=\left(6 / 5 \varepsilon^{2}\right)+13$ $\pi^{2}$. By Wirtinger's inequality and Sobolev inequality of Proposition 1.3 in [28], we have $\operatorname{ker}\left(A_{1}-\bar{B}_{0}\right)=R^{n},|x|=\|x\|_{\infty}=$ $\|x\|$ for all $x \in R^{n}$, and $\delta_{0}^{2}=1 / 12, \varepsilon_{2}^{2}=4 \pi^{2} /\left(1+4 \pi^{2}\right)$, $\left(\int_{0}^{1} f(t) d t\right)^{2}=((7 / 2 \pi)+(7 \varepsilon / 4))^{2}$ via a simple calculation.

Noticing that

$$
\begin{aligned}
\limsup _{|x| \rightarrow+\infty}|x|^{-2 \gamma_{0}} \int_{0}^{1} V(t, x) d t & =-\frac{3}{10}<-\frac{1+4 \pi^{2}}{96 \pi^{2}}\left(\frac{7}{2 \pi}+\frac{7 \varepsilon}{4}\right)^{2} \\
& =-\frac{\delta_{0}^{2}}{2 \varepsilon_{2}^{2}}\left(\int_{0}^{1} f(t) d t\right)^{2}
\end{aligned}
$$


as $\varepsilon>0$ sufficiently small, we know that $\left(H_{5}\right)$ holds. Set $\bar{B}_{01}$ $(t)=(2 \pi)^{2} I_{n}, \bar{B}_{02}(t)=4(2 \pi)^{2} I_{n}$. By $\bar{B}_{0}(t) \equiv 0$ and $V(t, x)=2$ $\pi^{2}\left(2+\sin ^{2} \pi t\right)|x|^{2}$ as $|x| \leq 1$, we can verify that $\left(H_{3}\right)$ holds. By Theorem 19, problem (89) has at least three distinct solutions. Obviously, Example 19 does not satisfy the conditions of Theorem 4 in [2] and Theorem 7 in [1].

Theorem 21. Assume that $V(t, x)$ satisfies $\left(H_{0}\right)$ and $\left(H_{6}\right) V(t, 0)=0$ for all $t \in[0,1]$

$\left(H_{7}\right)$ there exist $q(t) \in L^{1}\left([0,1], R^{+}\right)$and $\gamma \in(0,2)$ such that $V(t, x) \leq q(t)\left(1+|x|^{\gamma}\right)$ for all $x \in R^{n}$

$\left(H_{8}\right)$ there exist a positive constant $\tilde{c}$ and a $x_{0} \in \operatorname{ker}\left(A_{1}\right.$ $\left.-\bar{B}_{0}\right)$ with $\tilde{c}<\tilde{k}\left\|x_{0}\right\|_{L^{2}} \sqrt{\underline{\lambda}}$, such that

$$
\sup _{(t, x) \in[0,1] \times E_{1}} V(t, x)<\frac{\tilde{c}^{2}}{\bar{\lambda} \tilde{k}^{2}\left\|x_{0}\right\|_{L^{2}}^{2}} \int_{0}^{1} V\left(t, x_{0}(t)\right) d t
$$

where $E_{1}=\left\{x \in R^{n}|| x \mid \leq \tilde{c}\right\}$.

Then, there exist an open interval $\Lambda \subseteq[0,+\infty)$ and a positive number $r_{0}$ with the following property: for each $\eta \in \Lambda$ and for every $F(t, x):[0,1] \times R^{n} \longrightarrow R$ satisfies $\left(H_{0}\right)$, there exists $\rho>0$ such that, for each $\mu \in[0, \rho]$, problem (74) has at least three distinct solutions whose norms in $Z_{1}$ are less than $r_{0}$.

Proof. Clearly, $\left(\mathrm{H}_{6}\right)$ implies that $\Phi(\theta)=0$. Similar to the proof of Theorem 17, set

$$
\Psi(x)=\int_{0}^{1} F(t, x) d t, \forall x \in Z_{1}
$$

Then, $\left(\Psi_{0}\right)$ holds. Again set $X_{0}=L^{\infty}\left([0,1], R^{n}\right)$, we have $Z_{1} \subset X_{0} \subset X=L^{2}$ and the embeddings $Z_{1}^{\circ} X_{0}$ and $X_{0}^{\circ} X=L^{2}$ are compact and continuous, respectively. By $\left(\mathrm{H}_{7}\right)$, we obtain $\Phi(x) \leq \int_{0}^{1} q(t) d t+\|x\|_{\infty}^{\gamma} \int_{0}^{1} q(t) d t=c_{2}+c_{1}\|x\|_{X_{0}}^{\gamma}$ for all $x \in$ $Z_{1}$, which shows that $\left(\Phi_{7}\right)$ holds. Thanks to (81), we know that $E=\left\{x \in Z \mid\|x\|_{\infty} \leq \tilde{c}\right\} \subset E_{1}=\left\{x \in R^{n}|| x \mid \leq \tilde{c}\right\}$. So (15) follows from (104), which shows that $\left(\Phi_{8}\right)$ holds. Now, we can apply Theorem 3 to obtain Theorem 21 .

Remark 22. In particular, set $\bar{B}_{0}(t) \equiv 0$ and $M=I_{n}$. Then, the following problem

$$
\left\{\begin{array}{l}
-\ddot{x}+\bar{B}_{1}(t) x=\eta \nabla_{x} V(t, x)+\mu \nabla_{x} F(t, x), \quad \text { a.e. } t \in[0,1], \\
x(1)-x(0)=\dot{x}(1)-\dot{x}(0)=0,
\end{array}\right.
$$

has a similar conclusion of Theorem 21 , where $\tilde{k}=\sqrt{2}$. In addition, our result slightly generalizes Theorem 1 in [15] as $T=1, p=2$ in two aspects. At first, Theorem 21 requires $V(t, x)$ being measurable in $t$ for every $x \in R^{n}$ instead of continuous in $t$ for every $x \in R^{n}$; secondly Theorem 21 requires $\bar{B}_{1}(t) \in L^{\infty}\left([0,1], L_{s}\left(R^{n}\right)\right)$ instead of $\bar{B}_{1}(t) \in C\left([0,1], L_{s}\left(R^{n}\right)\right)$.

An example of problem (106) corresponding to Theorem 21 is given below.
Example 3. Let $n=2$,

$$
\bar{B}_{1}(t)=\left(\begin{array}{cc}
f_{1}(t) & 0 \\
0 & f_{1}(t)
\end{array}\right)
$$

and $f_{1}(t) \in L^{\infty}([0,1], R)$ with $\sup f_{1}(t)=2$, inf $f_{1}(t)=1$. Then, $\bar{\lambda}=2, \underline{\lambda}=1$. Let

$$
V\left(x_{1}, x_{2}\right)=\left(x_{1}^{2}+x_{2}^{2}\right)^{12} e^{1-\left(x_{1}^{2}+x_{2}^{2}\right)}+\left(x_{1}^{2}+x_{2}^{2}\right)^{\frac{3}{4}}, \forall\left(x_{1}, x_{2}\right) \in R^{2} .
$$

Then, for every $F(t, x):[0,1] \times R^{n} \longrightarrow R$ satisfies $\left(\mathrm{H}_{0}\right)$, all the hypotheses of Theorem 21 are satisfied with $\tilde{k}=\sqrt{2}, \tilde{c}=$ $1, x_{0}=(\sqrt{3}, \sqrt{6}), \gamma=3 / 2$ and $q(t)$ is a large enough constant. So, problem (106) has at least three distinct solutions. Noting that $f_{1}(t) \in C([0,1], R)$ is not necessarily true, we can see that the above example does not satisfy the conditions of Theorem 1 in [15].

Theorem 23. Assume that $x_{0}, \tilde{c}, \gamma, q(t)$, and $E_{1}$ are the same as in Theorem 21, $V(t, x)$ satisfies $\left(H_{0}\right),\left(H_{6}\right),\left(H_{7}\right)$, and $\left(H_{9}\right)$

$$
\sup _{(t, x) \in[0,1] \times E_{1}} V(t, x)<\frac{\tilde{c}^{2}}{\tilde{c}^{2}+\bar{\lambda} \tilde{k}^{2}\left\|x_{0}\right\|_{L^{2}}^{2}} \int_{0}^{1} V\left(t, x_{0}(t)\right) d t .
$$

Then, for each

$\eta \in\left(\frac{\bar{\lambda}\left\|x_{0}\right\|_{L^{2}}^{2}}{2\left(\int_{0}^{1} V\left(t, x_{0}(t)\right) d t-\sup _{(t, x) \in[0,1] \times E_{1}} V(t, x)\right)}, \frac{\tilde{c}^{2}}{2 \tilde{k}^{2} \sup _{(t, x) \in[0,1] \times E_{1}} V(t, x)}\right)=E_{2}$,

problem (74) admits at least three distinct solutions provided $\mu=0$.

Proof. Similar to the proof of Theorem 21, we need only to show that $\left(\Phi_{9}\right)$ follows from $\left(\mathrm{H}_{9}\right)$. Noticing that $E \subset E_{1}$, we have

$$
\sup _{x \in E} \Phi(x)=\sup _{x \in E} \int_{0}^{1} V(t, x) d t \leq \sup _{(t, x) \in[0,1] \times E_{1}} V(t, x)
$$

which means that $\left(\Phi_{9}\right)$ holds and

$$
\eta \in E_{2} \subset\left(\frac{\bar{\lambda}\left\|x_{0}\right\|_{L^{2}}^{2}}{2\left(\Phi\left(x_{0}\right)-\sup _{x \in E} \Phi(x)\right)}, \frac{\tilde{c}^{2}}{2 \tilde{k}^{2} \sup _{x \in E} \Phi(x)}\right) .
$$
23.

Therefore, we can apply Theorem 4 to obtain Theorem

Remark 24. In particular, set $\bar{B}_{0}(t) \equiv 0$ and $M=I_{n}$. Then, the following problem (106) has a similar conclusion of Theorem 
23. By the above Remark 5, we can see that our result slightly generalizes Theorem 2 of [15] as $T=1, p=2$ in two aspects.

Finally, we give an example of problem (106) corresponding to Theorem 23.

Example 4. Let $n=2$,

$$
\bar{B}_{1}(t)=\left(\begin{array}{cc}
f_{1}(t) & 0 \\
0 & f_{1}(t)
\end{array}\right)
$$

and $f_{1}(t) \in L^{\infty}([0,1], R)$ with $\sup f_{1}(t)=2$, inf $f_{1}(t)=1$. Then, $\bar{\lambda}=2, \underline{\lambda}=1$. Let

$$
V\left(x_{1}, x_{2}\right)=\frac{1}{9}\left(x_{1}^{2}+x_{2}^{2}\right)^{12} e^{1-\left(x_{1}^{2}+x_{2}^{2}\right)}, \forall\left(x_{1}, x_{2}\right) \in R^{2}
$$

Then, all the hypotheses of Theorem 23 are satisfied with $\tilde{k}=\sqrt{2}, \tilde{c}=1, x_{0}=(\sqrt{3}, \sqrt{6}), \gamma=3 / 2$ and $q(t)$ is a large enough constant. So, for each $\eta \in\left(81 e^{8} /\left(9^{12}-e^{8}\right), 9 / 4\right)$, problem (106) has at least three distinct solutions provided $\mu=0$. Noting that $f_{1}(t) \in C([0,1], R)$ is not necessarily true, we can see that the above example does not satisfy the conditions of Theorem 2 in [15].

4.2. Second-Order Hamiltonian Systems Satisfying SturmLiouville Boundary Value Conditions. As a second example, we discuss the solvability of the following two SturmLiouville boundary value problems

$$
\left\{\begin{array}{l}
-\ddot{x}-\tilde{B}_{0}(t) x=\nabla_{x} V(t, x), \text { a.e.t } \in[0,1], \\
x(0) \cos \alpha-\dot{x}(0) \sin \alpha=0, x(1) \cos \beta-\dot{x}(1) \sin \beta=0,
\end{array}\right.
$$

$\left\{\begin{array}{l}-\ddot{x}-\tilde{B}_{0}(t) x+\tilde{B}_{1}(t) x=\eta \nabla_{x} V(t, x)+\mu \nabla_{x} F(t, x), \text { a.e.t } \in[0,1], \\ x(0) \cos \alpha-\dot{x}(0) \sin \alpha=0, x(1) \cos \beta-\dot{x}(1) \sin \beta=0,\end{array}\right.$

where $\eta, \mu \in[0,+\infty), \tilde{B}_{0}(t), \tilde{B}_{1}(t) \in L^{\infty}\left([0,1], L_{s}\left(R^{n}\right)\right), \alpha \in[0$, $\pi), \beta \in(0, \pi]$, and $\nabla_{x} V(t, x)$ and $\nabla_{x} F(t, x)$ denote the gradient of $V(t, x)$ and $F(t, x)$ for $x \in R^{n}$, respectively.

Let $X=L^{2}\left([0,1], R^{n}\right)$. We define $A_{2}: D\left(A_{2}\right) \longrightarrow X$ by $\left(A_{2} x\right)(t)=-\ddot{x}(t) \quad$ where $\quad D\left(A_{2}\right)=\left\{x \in H^{2}\left([0,1], R^{n}\right) \mid x\right.$ satisfies $x(0) \cos \alpha-\dot{x}(0) \sin \alpha=0, x(1) \cos \beta-\dot{x}(1) \sin \beta=$ $0\}$. Set $\left(B_{i} x\right)(t)=\tilde{B}_{i}(t) x(t)(i=0,1)$. Then, $D\left(B_{i}\right)=X$. $A_{2}$ is self-adjoint and $\sigma\left(A_{2}\right)=\sigma_{d}\left(A_{2}\right)$ is bounded from below via Proposition 1.17 in [26]. Define $i_{\alpha, \beta}\left(\tilde{B}_{0}\right)=i_{A_{2}}\left(B_{0}\right), v_{\alpha, \beta}\left(\tilde{B}_{1}\right)$ $=v_{A_{2}}\left(B_{1}\right)$, that is, $v_{\alpha, \beta}\left(\tilde{B}_{0}\right)$ is the dimension of the solution subspace of (115) with $V(t, x) \equiv 0$.

Now, we assume that $i_{\alpha, \beta}\left(\tilde{B}_{0}\right)=0, v_{\alpha, \beta}\left(\tilde{B}_{0}\right) \neq 0, \tilde{B}_{1}(t)$ is positive definite for all $x \in R^{n}$, and $V, F:[0,1] \times R^{n} \longrightarrow R$ satisfies $\left(H_{0}\right)$. Meanwhile, set

$$
Z_{2}= \begin{cases}\left\{x \in H^{1}\left([0,1], R^{n}\right) \mid x(1)=0\right\}, & \alpha=0, \beta \in(0, \pi), \\ \left\{x \in H^{1}\left([0,1], R^{n}\right) \mid x(0)=0\right\}, & \alpha \in(0, \pi), \beta=\pi, \\ \left\{x \in H^{1}\left([0,1], R^{n}\right) \mid x(1)=x(0)=0\right\}, & \alpha=0, \beta=\pi, \\ H^{1}\left([0,1], R^{n}\right), & \alpha, \beta \in(0, \pi) .\end{cases}
$$

Thanks to Proposition 1.17 in [26], we have $Z_{2}=D$ $\left(\left|A_{2}\right|^{1 / 2}\right)$ and the norm

$$
\|x\|_{Z_{2}}=\left(\int_{0}^{1}\left[|\dot{x}(t)|^{2}+|x(t)|^{2}\right] d t\right)^{1 / 2}
$$

for each $x \in Z_{2}$. Noticing that $i_{\alpha, \beta}\left(\tilde{B}_{0}\right)=0, \nu_{\alpha, \beta}\left(\tilde{B}_{0}\right) \neq 0$, we can define another equivalent norm

$$
\|x\|=\left(\int_{0}^{1}\left[|\dot{x}(t)|^{2}-\left(\tilde{B}_{0}(t) x(t), x(t)\right)+|x(t)|^{2}\right] d t\right)^{1 / 2},
$$

for each $x \in Z_{2}$.

Let

$$
\begin{aligned}
\|x\|_{\tilde{B}_{1}}= & \left(\int _ { 0 } ^ { 1 } \left[|\dot{x}(t)|^{2}-\left(\tilde{B}_{0}(t) x(t), x(t)\right)\right.\right. \\
& \left.\left.+\left(\tilde{B}_{1}(t) x(t), x(t)\right)\right] d t\right)^{1 / 2}, \quad \forall x \in Z_{2} .
\end{aligned}
$$

Since $\tilde{B}_{1}(t) \in L^{\infty}\left([0,1], L_{s}\left(R^{n}\right)\right)$ is positive definite, there exist $\bar{\lambda}>\underline{\lambda}>0$ such that $\bar{\lambda}\|x\|_{L^{2}}^{2} \geq\left(B_{1} x, x\right)_{X} \geq \underline{\lambda}\|x\|_{L^{2}}^{2}$ for all $x \in X$. Similar to Section 4.1, we know that (80)-(83) hold. Hence, the following four results also hold. Because of the similarities with the proofs of Theorems 17, 19, 21, and 23, we omit them here.

Theorem 25. Assume that $V(t, x)$ satisfies $\left(H_{0}\right),\left(H_{1}\right)$, and $\left(\mathrm{H}_{2}\right)$ with $\bar{B}_{0}, \bar{B}_{2}$, and $\bar{B}_{3}$ replaced with $\tilde{B}_{0}, \tilde{B}_{2}$, and $\tilde{B}_{3}$, respectively, then problem (115) has at least one solution with saddle character in $Z_{2}$. In addition, assume that $V(t, x)$ satisfies $\left(H_{3}\right)$ with $\bar{B}_{01}$ and $\bar{B}_{02}$ replaced with $\tilde{B}_{01}$ and $\tilde{B}_{02}$, respectively, then problem (115) has at least three distinct solutions in $Z_{2}$.

Theorem 26. Assume that $V(t, x)$ satisfies $\left(H_{0}\right),\left(H_{4}\right)$, and $\left(H_{5}\right)$ with $\bar{B}_{0}$ replaced with $\tilde{B}_{0}$, then problem (115) has at least one solution in $Z_{2}$. In addition assume that $V(t, x)$ satisfies $\left(\mathrm{H}_{3}\right)$ with $\bar{B}_{01}$ and $\bar{B}_{02}$ replaced with $\tilde{B}_{01}$ and $\tilde{B}_{02}$, respectively, then problem (115) has at least three distinct solutions in $Z_{2}$.

Theorem 27. Assume that $V(t, x)$ satisfies $\left(H_{0}\right),\left(H_{6}\right),\left(H_{7}\right)$, and $\left(H_{8}\right)$ with $\bar{B}_{0}$ and $A_{1}$ replaced with $\tilde{B}_{0}$ and $A_{2}$, respectively, then there exist an open interval $\Lambda \subseteq[0,+\infty)$ and a positive number $r_{0}$ with the following property: for each $\eta \in \Lambda$ and 
for every $F(t, x):[0,1] \times R^{n} \longrightarrow R$ satisfies $\left(H_{0}\right)$, there exists $\rho>0$ such that, for each $\mu \in[0, \rho]$, problem (116) has at least three distinct solutions whose norms in $Z_{2}$ are less than $r_{0}$.

Theorem 28. Assume that $V(t, x)$ satisfies $\left(H_{0}\right),\left(H_{6}\right),\left(H_{7}\right)$, and $\left(H_{9}\right)$ with $\bar{B}_{0}$ and $A_{1}$ replaced with $\tilde{B}_{0}$ and $A_{2}$, respectively, then for each $\eta \in E_{2}$ problem (116) admits at least three distinct solutions provided $\mu=0$.

Remark 29. In particular, set $\alpha=0, \beta=\pi$ and $\tilde{B}_{0}(t) \equiv \pi^{2} I_{n}$. Then, we have $Z_{2}=H_{0}^{1}, \sigma\left(A_{2}\right)=\left\{k^{2} \pi^{2} \mid k \in N \backslash\{0\}\right\}$ and $\operatorname{ker}\left(A_{2}-\tilde{B}_{0}\right)=\left\{a \sin t \pi \mid a \in R^{n}\right\}$. Hence, the following problems

$$
\begin{gathered}
-\ddot{x}(t)-\pi^{2} x=\nabla_{x} V(t, x(t)), x(0)=x(1)=0, \\
\left\{\begin{array}{l}
-\ddot{x}-\pi^{2} x+\tilde{B}_{1}(t) x=\eta \nabla_{x} V(t, x)+\mu \nabla_{x} F(t, x), \text { a.e. } t \in[0,1], \\
x(0)=x(1)=0,
\end{array}\right.
\end{gathered}
$$

have the conclusion corresponding to Theorems 25-28, respectively, where $\tilde{B}_{01}(t) \equiv(k \pi)^{2} I_{n} \quad$ and $\tilde{B}_{02}(t)=$ $((k+1)) \pi)^{2} I_{n}$ with $k \geq 2$. In (122), if $\tilde{B}_{1}(t)=\pi^{2}, \mu=0$, then Theorem 27 corresponding to problem (122) reduces to Theorem 2 in [10] and Theorem 2 in [22], respectively, and our result requires $V(t, x)$ being measurable in $t$ for every $x \in R^{n}$ instead of continuous in $t$ for every $x \in R^{n}$.

\subsection{Elliptic Partial Differential Equations Satisfying Dirichlet} Boundary Value Conditions. As a third example, we discuss the solvability of the following two Dirichlet boundary value problems

$$
\begin{gathered}
\left\{\begin{array}{l}
-\Delta x-b_{0}(u) x=f(u, x), \quad u \in \Omega, \\
\left.x\right|_{\partial \Omega}=0,
\end{array}\right. \\
\left\{\begin{array}{l}
-\Delta x-b_{0}(u) x+b_{1}(u) x=\eta f(u, x)+\mu g(u, x), \quad u \in \Omega, \\
\left.x\right|_{\partial \Omega}=0,
\end{array}\right.
\end{gathered}
$$

where $\Omega \in R^{n}$ is a bounded open domain with smooth boundary, $\Delta x=\sum_{i=1}^{n}\left(\partial^{2} / \partial u_{i}^{2}\right) x, b_{0}(u), b_{1}(u) \in L^{\infty}(\Omega, R)$ and $\eta$ ,$\mu \in[0,+\infty)$. As usual, $\bar{\Omega}=\Omega \cup \partial \Omega$.

Let $X=L^{2}(\bar{\Omega}, R)$. We define $A_{3}: D\left(A_{3}\right) \longrightarrow X$ by $\left(A_{3} x\right)$ $(u)=-\Delta x(u)$ where $D\left(A_{3}\right)=H_{0}^{2}(\bar{\Omega})$. Set $\left(B_{i} x\right)(u)=b_{i}(u) x$ $(u)(i=0,1)$. Then, $D\left(B_{i}\right)=X$. It is well known that $A_{3}$ is self-adjoint and $\sigma\left(A_{1}\right)=\sigma_{d}\left(A_{1}\right) \subset[0,+\infty)$. Define $i_{\Delta}\left(b_{0}\right)$ $=i_{A_{3}}\left(B_{0}\right), v_{\Delta}\left(b_{0}\right)=v_{A_{3}}\left(B_{0}\right)$ (see Definition 2.5.1 in [23]), that is, $v_{\Delta}\left(b_{0}\right)$ is the dimension of the solution subspace of (123) as $f(u, x) \equiv 0$ and $i_{\Delta}\left(b_{0}\right)=\sum_{\lambda<0} v_{\Delta}\left(b_{0}+\lambda\right)$.

Now, we assume that $b_{1}(u)$ is positive definite for all $x \in R$, that is, there exists a positive constant $\underline{\lambda}$ such that $b_{1}(u) x^{2} \geq \underline{\lambda} x^{2}$ for all $x \in R$, and two Carathe' odory functions $f, g: \Omega \times R \longrightarrow R$ satisfy the following condition:
$\left(P_{0}\right)$ there exist $a_{i}>0(i=1,2)$ such that

$$
\begin{aligned}
& |f(u, x)| \leq a_{1}\left(1+|x|^{s_{1}}\right), \forall(u, x) \in \Omega \times R, \\
& |g(u, x)| \leq a_{2}\left(1+|x|^{s_{2}}\right), \forall(u, x) \in \Omega \times R,
\end{aligned}
$$

where $s_{1}, s_{2} \in(0,(n+2) /(\mathrm{n}-2))$ if $n>2$ and $s_{1}, s_{2} \in(0,+\infty)$ if $n=2$.

Further, assume that $i_{\Delta}\left(b_{0}\right)=0, v_{\Delta}\left(b_{0}\right) \neq 0$. Meanwhile, set $Z_{3}=H_{0}^{1}(\bar{\Omega})=W_{0}^{1,2}(\Omega)$. Obviously, $Z_{3}=D\left(\left|A_{3}\right|^{1 / 2}\right)$ and the norm

$$
\|x\|_{Z_{3}}=\left(\int_{\Omega}\left[|\nabla x(u)|^{2}+x^{2}(u)\right] d u\right)^{1 / 2}
$$

for each $x \in Z_{3}$. Noticing that $i_{\Delta}\left(b_{0}\right)=0, v_{\Delta}\left(b_{0}\right) \neq 0$, we can define another equivalent norm

$$
\|x\|=\left(\int_{\Omega}\left[|\nabla x(u)|^{2}-b_{0}(u) x^{2}(u)+x^{2}(u)\right] d u\right)^{1 / 2} \text {, }
$$

for each $x \in Z_{3}$.

Let

$\|x\|_{b_{1}}=\left(\int_{\Omega}\left[|\nabla x(u)|^{2}-b_{0}(u) x^{2}(u)+b_{1}(u) x^{2}(u)\right] d u\right)^{1 / 2}, \quad \forall x \in Z_{3}$.

Since $b_{1}(u) \in L^{\infty}(\Omega, R)$ is positive definite, there exist $\bar{\lambda}$ $>\underline{\lambda}>0$ such that $\bar{\lambda}\|x\|_{L^{2}}^{2} \geq \int_{\Omega} b_{1}(u) x^{2}(u) d u \geq \underline{\lambda}\|x\|_{L^{2}}^{2}$ for all $x \in X$. So we have

$$
\min \{1, \underline{\lambda}\}\|x\|^{2} \leq\|x\|_{b_{1}}^{2} \leq \max \{1, \bar{\lambda}\}\|x\|^{2} .
$$

Observe that the embedding $Z_{3}^{\hookrightarrow} L^{2}=X$ is compact. Let $\|\cdot\|_{\infty}$ be the norm of $L^{\infty}(\Omega, R)$. Then, there is a constant $\delta_{0}>0$ such that

$$
|x| \leq\|x\|_{\infty} \leq \delta_{0}\|x\|
$$

for any $x \in Z_{3}$. Let

$$
k_{2}=\sup _{x \in Z_{3} \backslash\{\theta\}} \frac{\|x\|_{\infty}}{\|x\|_{b_{1}}} .
$$

From (129) and (130), we have $0<k_{2} \leq \tilde{k}=\delta_{0}$ $(\min \{1, \underline{\lambda}\})^{-1 / 2}$.

For any $b_{1}(u), b_{2}(u) \in L^{\infty}(\Omega, R)$, we write $b_{1} \leq b_{2}$ if $b_{1}(u) \leq b_{2}(u)$ for a.e. $u \in \Omega$ and define $b_{1}<b_{2}$ if $b_{1}(u)<$ $b_{2}(u)$ a.e. $u \in \Omega$. From Theorems $1-4$, we have the following four results hold.

Theorem 30. Assume that $f(u, x)$ satisfies $\left(P_{0}\right)$ and 
$\left(P_{1}\right)$ there exist $g \in L^{1}\left(\Omega, R^{+}\right)$and $b_{2} \in L^{\infty}(\Omega, R)$ with $b_{2}$ $\geq 0, v_{\Delta}\left(b_{0}+b_{2}\right)=0$ and $i_{\Delta}\left(b_{0}+b_{2}\right)=v_{\Delta}\left(b_{0}\right)$ such that

$$
|f(u, x)| \leq b_{2}(u)|x|+g(u),
$$

for all $x \in R$ and a.e. $u \in \Omega$

$\left(P_{2}\right)$ there exists a positive definite $b_{3} \in L^{\infty}(\Omega, R)$ such that $\int_{0}^{x} f(u, t) d t-(1 / 2) b_{3}(u) x^{2}$ is convex in $x$ for a.e. $u \in \Omega$

Then, problem (123) has at least one solution with saddle character in $Z_{3}$.

Assume in addition that

$\left(P_{3}\right)$ there exist $\left.\delta_{1}>0, b_{01}, b_{02} \in L^{\infty}(\Omega, R)\right)$ such that $b_{02}>b_{01}>b_{0}$ and $v_{\Delta}\left(b_{0 i}\right) \neq 0(i=1,2), \quad i_{\Delta}\left(b_{02}\right)=i_{\Delta}\left(b_{01}\right)+$ $v_{\Delta}\left(b_{01}\right)$, and for all $|x| \leq \delta_{1}$,

$$
\frac{1}{2}\left(b_{01}(u)-b_{0}(u)\right) x^{2} \leq \int_{0}^{x} f(u, t) d t \leq \frac{1}{2}\left(b_{02}(u)-b_{0}(u)\right) x^{2},
$$

for a.e. $u \in \Omega$. Then, problem (123) has at least three distinct solutions in $Z_{3}$.

Proof. Set

$$
\left.\Phi(x)=\int_{\Omega}\left(\int_{0}^{x} f(u, t) d t\right)\right) d u, \quad \forall x \in Z_{3} .
$$

Then, $\Phi \in C^{1}\left(Z_{3}, R\right)$ is weakly continuous with compact derivative, and for every $x \in Z_{3}$,

$$
\Phi^{\prime}(x) y=\int_{\Omega} f(u, x) y d u, \quad \forall y \in Z_{3},
$$

because of $\left(\mathrm{P}_{0}\right)$ and (132). Hence, $\left(\Phi_{0}\right)$ holds.

Thanks to (130) and (132) of $\left(P_{1}\right)$, we have

$$
\begin{aligned}
\left|(\nabla \Phi(x), x)_{L^{2}}\right| & \leq \int_{\Omega} b_{2}(u) x^{2}(u) d u+\int_{\Omega} g(u)|x(u)| d u \\
& \leq\left(B_{2} x, x\right)_{L^{2}}+\|x\|_{\infty} \int_{\Omega} g(u) d u \\
& \leq\left(B_{2} x, x\right)_{L^{2}}+c_{0}\|x\|,
\end{aligned}
$$

for all $x \in L^{2}$, where $c_{0}=\delta_{0} \int_{\Omega} g(u) d u$. This shows that $\left(\Phi_{1}\right)$ holds.

Next, similar to the proof of Theorem 17, we can get the conclusion of Theorem 30 via Theorem 1.

Theorem 31. Assume that $f(u, x)$ satisfies $\left(P_{0}\right)$ and

$\left(P_{4}\right)$ there exist $f_{1}, g \in L^{1}\left(\Omega, R^{+}\right)$and $\gamma_{0} \in(0,1)$ such that

$$
|f(u, x)| \leq f_{1}(u)|x|^{\gamma_{0}}+g(u),
$$

for all $x \in R$ and a.e. $u \in \Omega$
$\left(P_{5}\right)$ there exists $c_{3}>\left(\delta_{0}^{2} / 2 \varepsilon_{2}^{2}\right)\left(\int_{\Omega} f(u) d u\right)^{2}>0$ such that

$$
\limsup _{\|x\| \rightarrow+\infty}\|x\|^{-2 \gamma_{0}} \int_{\Omega}\left(\int_{0}^{x} f(u, t) d t\right) d u<-c_{3}
$$

for all $x \in \operatorname{ker}\left(A_{3}-b_{0}\right)$, where the constants $\delta_{0}$ and $\varepsilon_{2}$ are given by (130) and (49), respectively.

Then, problem (123) has at least one solution in $Z_{3}$. Further, assume that $f(u, x)$ satisfies $\left(P_{3}\right)$. Then, problem (123) has at least three distinct solutions in $Z_{3}$.

Proof. Similar to the proof of Theorem 30, we need only to show that $\left(\Phi_{4}\right)$ follows from $\left(P_{4}\right)$. By $\left(P_{4}\right)$, we have

$$
\begin{aligned}
\left|(\nabla \Phi(x), y)_{L^{2}}\right| & \leq \int_{\Omega} f_{1}(u)|x(u)|^{\gamma_{0}} \cdot|y(u)| d t+\int_{\Omega} g(u)|y(u)| d u \\
& \leq c_{1}\|x\|_{\infty}^{\gamma_{0}} \cdot\|y\|_{\infty}+c_{2}\|x\|_{\infty},
\end{aligned}
$$

for all $x, y \in L^{2}$, where $c_{1}=\int_{\Omega} f_{1}(u) d u, c_{2}=\int_{\Omega} g(u) d u$, which shows that $\left(\Phi_{4}\right)$ holds. So, Theorem 31 follows from Theorem 2.

Theorem 32. Assume that $f(u, x)$ satisfies $\left(P_{0}\right)$ and

$\left(P_{7}\right)$ there exist $q(u) \in L^{1}\left(\Omega, R^{+}\right)$and $\gamma \in(0,2)$ such that $\int_{0}^{x} f(u, t) d t \leq q(u)\left(1+|x|^{\gamma}\right)$ for all $x \in R$

$\left(P_{8}\right)$ there exist a positive constant $\tilde{c}$ and $a x_{0} \in \operatorname{ker}\left(A_{3}-\right.$ $\left.b_{0}\right)$ with $\tilde{c}<\tilde{k}\left\|x_{0}\right\|_{L^{2}} \sqrt{\underline{\lambda}}$, such that

$$
\sup _{(u, x) \in \Omega \times E_{1}} \int_{0}^{x} f(u, t) d t<\frac{\tilde{c}^{2}}{\bar{\lambda} \tilde{k}^{2}\left\|x_{0}\right\|_{L^{2}}^{2} \cdot \operatorname{meas}(\Omega)} \int_{\Omega} \int_{0}^{x_{0}} f(u, t) d t d u,
$$

where $E_{1}=\{x \in R|| x \mid \leq \tilde{c}\}$.

Then, there exist an open interval $\Lambda \subseteq[0,+\infty)$ and a positive number $r_{0}$ with the following property: for each $\eta \in \Lambda$ and for every $g(u, x): \Omega \times R \longrightarrow R$ satisfies $\left(P_{0}\right)$, there exists $\rho>0$ such that, for each $\mu \in[0, \rho]$, problem (124) has at least three distinct solutions whose norms in $Z_{3}$ are less than $r_{0}$.

Proof. Clearly, $\Phi(\theta)=0$ via the definition of $\Phi(x)$. Set $X_{0}=$ $L^{\infty}(\Omega, R)$, we have $Z_{3} \subset X_{0} \subset X=L^{2}$ and the embeddings $Z_{3}{ }^{\circ} X_{0}$ and $X_{0}^{\circ} X=L^{2}$ are compact and continuous, respectively. Similar to the proofs of Theorem 21 and Theorem 30 , we can get the conclusion of Theorem 32 via Theorem 3 .

Remark 33. As $p=2$ in [9], Theorem 32 reduces to Theorem 2.4 in [9], and the continuity of $f(u, x)$ in $u$ and the condition (i) of Theorem 2.4 in [9] is discarded. 
Theorem 34. Assume that $x_{0}, \tilde{c}, \gamma, q(u)$, and $E_{1}$ are the same as in Theorem 32, $f(u, x)$ satisfies $\left(P_{0}\right),\left(P_{7}\right)$, and $\left(P_{9}\right)$

$\sup _{(u, x) \in \Omega \times E_{1}} \int_{0}^{x} f(u, t) d t<\frac{\tilde{c}^{2}}{\left(\tilde{c}^{2}+\bar{\lambda} \tilde{k}^{2}\left\|x_{0}\right\|_{L^{2}}^{2}\right) \cdot \operatorname{meas}(\Omega)} \int_{\Omega} \int_{0}^{x_{0}} f(u, t) d t d u$.

Then, for each

$$
\eta \in\left(\frac{\bar{\lambda}\left\|x_{0}\right\|_{L^{2}}^{2}}{2 \rho_{0}}, \frac{\tilde{c}^{2}}{2 \tilde{k}^{2} \rho_{1}}\right)=E_{2}
$$

problem (124) admits at least three distinct solutions provided $\mu=0$, where

$$
\begin{aligned}
& \rho_{0}=\int_{\Omega} \int_{0}^{x_{0}} f(u, t) d t d u-\rho_{1}, \\
& \rho_{1}=\operatorname{meas}(\Omega) \sup _{(u, x) \in \Omega \times E_{1}} \int_{0}^{x} f(u, t) d t .
\end{aligned}
$$

Proof. Similar to the proof of Theorem 32, we need only to show that $\left(\Phi_{9}\right)$ follows from $\left(P_{9}\right)$. Noticing that $E \subset E_{1}$, we have

$$
\sup _{x \in E} \Phi(x)=\sup _{x \in E} \int_{\Omega} \int_{0}^{x} f(u, t) d t d u \leq \operatorname{meas}(\Omega) \sup _{(u, x) \in \Omega \times E_{1}} \int_{0}^{x} f(u, t) d t,
$$

which means that $\left(\Phi_{9}\right)$ holds and

$$
\eta \in E_{2} \subset\left(\frac{\bar{\lambda}\left\|x_{0}\right\|_{L^{2}}^{2}}{2\left(\Phi\left(x_{0}\right)-\sup _{x \in E} \Phi(x)\right)}, \frac{\tilde{c}^{2}}{2 \tilde{k}^{2} \sup _{x \in E} \Phi(x)}\right) .
$$
34.

Therefore, we can apply Theorem 4 to obtain Theorem

\section{Data Availability}

No data were used to support this study.

\section{Conflicts of Interest}

The authors declare that they have no conflicts of interest.

\section{Authors' Contributions}

All authors typed, read, and approved the final manuscript.

\section{Acknowledgments}

This research was supported by the National Natural Science Foundation of China (No. 12071219).

\section{References}

[1] H. Brezis and L. Nirenberg, "Remarks on finding critical points," Communications on Pure and Applied Mathematics, vol. 44, pp. 939-963, 1991.

[2] C. L. Tang, "Periodic solutions for nonautonomous second order systems with sublinear nonlinearity," Proceedings of the American Mathematical Society, vol. 126, no. 11, pp. 32633270, 1998.

[3] X. Wu, "Saddle point characterization and multiplicity of periodic solutions of nonautonomous second-order systems," Nonlinear Analysis, vol. 58, pp. 899-907, 2004.

[4] H. Amann, "Saddle points and multiple solutions of differential equations," Mathematische Zeitschrift, vol. 169, pp. 127166, 1979.

[5] B. Ricceri, "On a three critical points theorem," Archiv der Mathematik, vol. 75, no. 3, pp. 220-226, 2000.

[6] B. Ricceri, "A three critical points theorem revisited," Nonlinear Analysis, vol. 70, no. 9, pp. 3084-3089, 2009.

[7] R. P. Agarwal, A. M. Alghamdi, S. Gala, and M. A. Ragusa, “On the continuation principle of local smooth solution for the Hall-MHD equations," Applicable Analysis, pp. 1-9, 2020.

[8] R. P. Agarwal, S. Gala, and M. A. Ragusa, "A regularity criterion in weak spaces to Boussinesq equations," Mathematics, vol. 8, no. 6, p. 920, 2020.

[9] G. A. Afrouzi and S. Heidarkhani, "Three solutions for a Dirichlet boundary value problem involving the p-Laplacian," Nonlinear Analysis, vol. 66, pp. 2281-2288, 2007.

[10] G. Bonanno, "Existence of three solutions for a two point boundary value problem," Applied Mathematics Letters, vol. 13, pp. 53-57, 2000.

[11] G. Bonanno and R. Livrea, "Multiplicity theorems for the Dirichlet problem involving the p-Laplacian," Nonlinear Analysis, vol. 54, pp. 1-7, 2003.

[12] C. Li and C. L. Tang, "Three solutions for a class of quasilinear elliptic systems involving the (p,q)-Laplacian," Nonlinear Analysis, vol. 69, pp. 3322-3329, 2008.

[13] G. Bonanno and R. Livrea, "Periodic solutions for a class of second-order Hamiltonian systems," Electronic Journal of Differential Equations, vol. 115, pp. 1-13, 2005.

[14] D. Averna and G. Bonanno, "A three critical point theorem and its applications to the ordinary Dirichlet problem," Topological Methods in Nonlinear Analysis, vol. 22, pp. 93-103, 2003.

[15] C. Li, Z. Q. Ou, and C. L. Tang, "Three periodic solutions for pHamiltonian systems," Nonlinear Analysis, vol. 74, pp. 15961606, 2001.

[16] K. C. Chang, "Solutions of asymptotically linear operator equations via Morse theory," Communications on Pure and Applied Mathematics, vol. 34, pp. 693-712, 1981.

[17] K. C. Chang, Infinite Dimensional Morse Theory and Multiple Solution Problems, Birkhäuser, Boston, MA, 1993.

[18] K. C. Chang, J. Q. Liu, and M. J. Liu, "Nontrivial periodic solutions for strong resonance Hamiltonian systems," Annales de l'Institut Henri Poincare-Non Linear Analysis., vol. 14, no. 1, pp. 103-117, 1997.

[19] K. C. Chang, S. P. Wu, and S. Li, "Multiple periodic solutions for an asymptotically linear wave equation," Indiana University Mathematics Journal, vol. 31, pp. 721-731, 1982.

[20] M. Struwe, "A note on a result of Ambrosetti and Mancini," Annali di Matematica Pura ed Applicata, vol. 131, no. 1, pp. 107-115, 1982. 
[21] V. C. Zelati, I. Ekeland, and E. Séré, "A variational approach to homolinic orbits in Hamiltonian systems," Mathematische Annalen, vol. 288, no. 1, pp. 133-160, 1990.

[22] P. Candito, "Existence of three solutions for a nonautonomous two point boundary value problem," Journal of Mathematical Analysis and Applications, vol. 252, pp. 532-537, 2000.

[23] Y. J. Dong, "Index theory for linear self-adjoint operator equations and nontrivial solutions for asymptotically linear operator equations," Calculus of Variations and Partial Differential Equations, vol. 38, pp. 75-109, 2010.

[24] Y. J. Dong, Index Theory for Hamiltonian Systems and Multiple Solutions Problems, Science Press, Beijing, 2014.

[25] B. Ricceri, "Existence of three solutions for a class of elliptic eigenvalue problems," Mathematical and Computer Modelling, vol. 32, no. 11-13, pp. 1485-1494, 2000.

[26] Y. Chen, Y. Dong, and Y. Shan, "Existence of solutions for sublinear or superlinear operator equations," Science China. Mathematics, vol. 58, pp. 1653-1664, 2015.

[27] M. L. Song, "Existence of solutions for subquadratic convex or B-concave operator equations and applications to second order Hamiltonian systems," Electronic Journal of Qualitative Theory of Differential Equations, vol. 49, pp. 1-19, 2020.

[28] J. Mawhin and M. Willem, Critical Point Theory and Hamiltonian Systems, Springer, Berlin, 1989.

[29] E. Zeidler, Nonlinear Functional Analysis and Its Applications, Vol. II/B, Springer, Berlin, Heidelberg, New York, 1985.

[30] I. Ekeland, Convexity Methods in Hamiltonian Mechanics, Springer-Verlag, Berlin, 1990.

[31] P. H. Rabinowitz, "Variational methods for Hamiltonian systems," in Handbook of Dynamical Systems, vol. 1, pp. 10911127, Elsevier, 2002.

[32] R. Iannacci, M. Nkashama, and J. Ward, "Nonlinear second order elliptic partial differential equations at resonance," Transactions of the American Mathematical Society, vol. 311, no. 2, pp. 711-726, 1989.

[33] R. Iannacci and M. Nkashama, "Nonlinear elliptic partial differential equations at resonance: higher eigenvalues," Nonlinear Analysis, vol. 25, pp. 455-471, 1995.

[34] P. H. Rabinowitz, Minimax Methods in Critical Point Theory with Application to Differential Equations, CBMS Regional Conference Series in Mathematics, vol. 65, Amer. Math. Soc, Providence, RI, 1986. 